\title{
PIERRE FABRIE
}

MAZEN SAAD

\section{Existence de solutions faibles pour un modèle d'écoulement triphasique en milieu poreux}

Annales de la faculté des sciences de Toulouse $6^{e}$ série, tome $2, \mathrm{n}^{\circ} 3$ (1993), p. 337-373

<http://www.numdam.org/item?id=AFST_1993_6_2_3_337_0>

(C) Université Paul Sabatier, 1993, tous droits réservés.

L'accès aux archives de la revue «Annales de la faculté des sciences de Toulouse » (http://picard.ups-tlse.fr/ annales/) implique l'accord avec les conditions générales d'utilisation (http://www.numdam.org/conditions). Toute utilisation commerciale ou impression systématique est constitutive d'une infraction pénale. Toute copie ou impression de ce fichier doit contenir la présente mention de copyright.

\section{NumDam}

Article numérisé dans le cadre du programme

Numérisation de documents anciens mathématiques

http://www.numdam.org/ 


\title{
Existence de solutions faibles pour un modèle d'écoulement triphasique en milieu poreux ${ }^{(*)}$
}

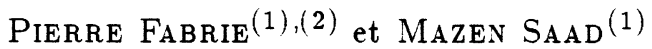

\begin{abstract}
RÉSUMÉ. - La modélisation des écoulements triphasiques incompressibles en milieu poreux homogène donne lieu à un système d'équations aux dérivées partielles semi-linéaires de type elliptique parabolique dégénéré. Le but de cet article est de montrer que, sous une certaine condition liant les perméabilités relatives, le problème régularisé est bien posé. Nous explicitons ensuite une condition suffisante pour que le problème dégénéré soit lui aussi bien posé.
\end{abstract}

Abstract. - Three-phase incompressible flows in homogeneous porous media are governed by a system of semilinear elliptic-parabolic degenerate partial differential equations. The goal of this paper is to show that under the total differentiability condition of G. Chavent, the regularised problem is well posed. We then give explicit sufficient conditions which make the degenerate problem well posed.

\section{Introduction}

On s'intéresse ici à la modélisation de l'écoulement de trois phases lors de l'exploitation d'un gisement pétrolier à un seul type de roche. Les techniques de récupération du pétrole consistent à déplacer les fluides présents dans le milieu vers les puits de production en injectant de l'eau par des puits réservés à cet effet.

(*) Reçu le 17 juin 1992

(1) CeReMaB - U.R.A. 226, 351 Cours de la Libération, 33405 Talence Cedex (France)

(2) L.E.P.T. - ENSAM, U.R.A. 873, Esplanade des Arts et Métiers, 33405 Talence Cedex (France) 
On propose ici une étude théorique du système modélisant les écoulements triphasiques incompressibles immiscibles isothermes en milieu poreux homogène. Hormis la phase eau, les deux autres phases désignent deux composants liquides hydrocarburés non miscibles et sans interaction chimique. Le système d'équations aux dérivées partielles que l'on est amené à étudier est un système parabolique dégénéré couplé à une équation elliptique. Les travaux de G. Chavent [6]-[7] décrivent un modèle mathématique pour l'étude théorique de ce type de déplacements.

Plusieurs auteurs se sont intéressés aux modèles de déplacements forcés des écoulements diphasiques incompressibles et ils ont établi l'existence d'une solution faible pour ces types d'écoulements sous l'hypothèse de différentiabilité totale de G. Chavent. Citons par exemple G. Gagneux [11], B. Amaziane [3], T. Arbogast [4], H. W. Alt et E. Di Benedetto [1], G. Chavent [6]-[7], U. Hornung [15].

Bien que les modèles triphasiques incompressibles soient très étudiés numériquement, ils ont donné lieu à peu d'études théoriques. En particulier, nous ne connaissons pas de résultats où il est montré que l'un des modèles couramment utilisé en génie pétrolier conduit à un système bien posé.

En premier lieu, on établit un résultat d'existence pour le système régularisé par la méthode de viscosité artificielle. Pour cela, on découple le système obtenu en introduisant un retard positif en temps. On montre ensuite la stabilité du schéma en établissant des estimations a priori pour les solutions approchées. Celle-ci assurent la convergence, vers la solution du problème régularisé, lorsque le retard tend vers zéro. Enfin on montre que la solution est physiquement admissible, c'est-à-dire que les saturations sont positives et de somme égale à un. En second lieu, sous une condition liant les perméabilités relatives, on établit un résultat d'existence pour le système global modélisant les écoulements triphasiques incompressibles, par convergence des solutions du problème régularisé.

\section{Modèle physique}

On s'intéresse au problème de la récupération du pétrole d'un gisement constitué d'un seul type de roche caractérisé par les perméabilités intrinsèques, les pressions capillaires et les perméabilités relatives.

La technique utilisée est celle de déplacements forcés de fluides par un autre. On considère que le fluide est constitué de trois constituants, l'eau et 
deux composants liquides non miscibles et sans interaction chimique. Ces deux constituants seront notés abusivement "phase huile" et "phase gaz" par analogie avec les écoulements triphasiques à une phase compressible.

On désigne par $\Omega$ un ouvert borné de $\mathbb{R}^{3}$, de frontière $\Gamma$ régulière. Soit $n$ le vecteur normal extérieur à $\Gamma$ et $[0, T]$ l'intervale du temps d'étude.

La frontière $\Gamma$ est partitionnée comme suit :

$$
\Gamma=\Gamma_{e} \cup \Gamma_{i} \cup \Gamma_{s}, \quad \Gamma_{i} \cap \Gamma_{j}=\emptyset, i \neq j
$$

avec

$\Gamma_{e}$ frontière d'injection de l'eau,

$\Gamma_{s}$ frontière de récupération de l'huile et du gaz,

$\Gamma_{i}$ frontière imperméable.

On note :

$\Sigma_{j}=\Gamma_{j} \times[0, T[, j=e, s, i ;$

$w$ la phase eau

$o$ la phase huile

$g$ la phase gaz

$s_{\eta}$ la saturation de la phase $\eta, \eta=w, g, o$

$V_{\eta}$ la vitesse de la phase $\eta, \eta=w, g, o$

$\Phi(x)$ la porosité

$\mathbf{K}(x)$ la perméabilité intrinsèque. Elle dépend de la nature du milieu et de sa géométrie

$K_{\eta}=K_{\eta}\left(s_{w}, s_{g}\right)$ la perméabilité relative de la phase $\eta, \eta=w, o, g$

$p_{\eta}$ la pression de la phase $\eta, \eta=w, o, g$

$\mu_{\eta}$ la viscosité de la phase $\eta, \eta=w, o, g$

$\rho_{\eta}$ la masse volumique de la phase $\eta, \eta=w, o, g$

$g$ le vecteur accélération de la pesanteur

$p_{c w}=p_{c w}\left(s_{w}, s_{g}\right)$ la pression capillaire de l'eau par rapport à celle de l'huile

$p_{c g}=p_{c g}\left(s_{w}, s_{g}\right)$ la pression capillaire du gaz par rapport à celle de l'huile.

Les équations modélisant les déplacements triphasiques immiscibles, faiblement compressibles (on néglige les variations de la pression de chaque phase en fonction de la densité) sont alors les suivantes :

Conservation du volume. - Elle s'écrit pour chaque constituant

$$
\partial_{t}\left(\boldsymbol{\Phi} s_{\eta}\right)+\operatorname{div} V_{\eta}=0, \quad \eta=w, o, g .
$$


On considère sans perte de généralité que la saturation irréductible de chaque phase est nulle.

On a de plus

$$
s_{w}+s_{g}+s_{o}=1
$$

Conservation de la quantité de mouvement. - Elle se traduit par la loi de Darcy

$$
V_{\eta}=-\mathbf{K}(x) \frac{K_{\eta}\left(s_{w}, s_{g}\right)}{\mu_{\eta}}\left[\nabla p_{\eta}-\rho_{\eta} g\right] \quad \text { pour } \eta=w, o, g .
$$

La pression capillaire est la différence de pression entre deux phases dans les pores. Il est supposé que l'eau est la phase mouillante dans le modèle eau-huile et que l'huile est la phase mouillante dans le modèle huile-gaz. On définit alors deux pressions capillaires

$$
\begin{aligned}
p_{c w} & =p_{w}-p_{o} \\
p_{c g} & =p_{g}-p_{o}
\end{aligned}
$$

On considère dans cette étude, conformément à [1] et [7] que la saturation $s_{\eta}, \eta=w, g$ est une fonction des pressions capillaires $p_{c w}$ et $p_{c g}$ de même la perméabilité relative $K_{\eta}, \eta=w, g$, ost une fonction des saturations $s_{w}$ et $s_{g}$. Le comportement qualitatif de ces fonctions est obtenu par mesures expérimentales.

Nous allons maintemant préciser les propriétés essentielles des fonctions $s_{w}$ et $s_{g}$, les fonctions $p_{c g}$ et $p_{c w}$ étant supposées indépendantes :

i) $s_{w}\left(\boldsymbol{p}_{c}\right)=s_{w}\left(p_{c w}, p_{c g}\right), s_{g}\left(\boldsymbol{p}_{c}\right)=s_{g}\left(p_{c w}, p_{c g}\right)$;

ii) Les saturations sont des fonctions de classe $C^{1}$ sur un voisinage ouvert de $[-1,0] \times[0,1]$ et vérifient :

- $\exists\left(s_{\eta}^{1}, s_{\eta}^{2}\right) \in \mathbb{R}_{+}^{*} \times \mathbb{R}_{+}^{*}$ tel que

$$
0<s_{\eta}^{1}<\frac{\partial s_{\eta}}{\partial p_{c \eta}}\left(p_{c}\right)<s_{\eta}^{2}, \forall p_{c} \in[-1,0] \times[0,1], \eta=w, g
$$

- $\exists\left(s_{\eta}^{3}, s_{\eta}^{4}\right) \in \mathbb{R}_{-}^{*} \times \mathbb{R}_{-}^{*}$ tel que

$$
\begin{array}{r}
\boldsymbol{s}_{\eta}^{3}<\frac{\partial s_{\eta}}{\partial p_{c \mu}}\left(\boldsymbol{p}_{c}\right)<\boldsymbol{s}_{\eta}^{4}<0, \forall \boldsymbol{p}_{c} \in[-1,0] \times[0,1], \\
(\eta, \mu)=(w, g) \text { ou }(g, w) ;
\end{array}
$$


iii) $s_{w}\left(-1, p_{c g}\right)=0, s_{w}\left(0, p_{c g}\right)=1, \forall p_{c g} \in[0,1]$

$s_{g}\left(p_{c w}, 0\right)=0, s_{g}\left(p_{c w}, 1\right)=1, \forall p_{c w} \in[-1,0]$;

iv) $\frac{\partial \boldsymbol{s}_{w}}{\partial p_{c w}}\left(\boldsymbol{p}_{c}\right)>\left|\frac{\partial \boldsymbol{s}_{w}}{\partial p_{c g}}\left(\boldsymbol{p}_{c}\right)\right|, \frac{\partial s_{g}}{\partial p_{c g}}\left(\boldsymbol{p}_{c}\right)>\left|\frac{\partial \boldsymbol{s}_{g}}{\partial p_{c w}}\left(\boldsymbol{p}_{c}\right)\right|$, $\forall \boldsymbol{p}_{c} \in[-1,0] \times[0,1]$;

v) $\frac{\partial \boldsymbol{s}_{w}}{\partial p_{c g}}\left(\boldsymbol{p}_{c}\right)=\frac{\partial \boldsymbol{s}_{g}}{\partial p_{c w}}\left(\boldsymbol{p}_{c}\right), \forall \boldsymbol{p}_{c} \in[-1,0] \times[0,1]$.

On regroupe par la suite les hypothèses i), ii), iii), iv) et v) sous la rubrique hypothèse $(\mathrm{H} 1)$. Ces hypothèses sont physiquement réalistes.

\section{Remarques 1}

1) Les hypothèses iv) et $\mathbf{v}$ ) permettent de définir une fonction $\Theta$ de $\mathbb{R}^{2}$ dans $\mathbb{R}$, de classe $C^{2}$, strictement convexe telle que

$$
\boldsymbol{s}\left(\boldsymbol{p}_{c}\right)={ }^{t}\left(s_{w}\left(\boldsymbol{p}_{c}\right), \boldsymbol{s}_{g}\left(\boldsymbol{p}_{c}\right)\right)=\boldsymbol{\nabla} \Theta\left(\boldsymbol{p}_{c}\right)
$$

et vérifiant de plus

$$
\exists \theta \in \mathbb{R}_{+}^{*} \text { tel que } \Theta^{\prime \prime}(\boldsymbol{y}) \cdot \boldsymbol{p}_{c} \cdot \boldsymbol{p}_{c} \geq \theta\left|\boldsymbol{p}_{c}\right|^{2}, \quad \forall \boldsymbol{y} \in \mathbb{R}^{2} .
$$

De façon analogue à [2], on définit alors la fonction

$$
\psi(\boldsymbol{z})=\sup _{\boldsymbol{\sigma} \in \mathbb{R}^{2}}(\boldsymbol{z} \cdot \boldsymbol{\sigma}-\Theta(\boldsymbol{\sigma})+\Theta(-1,0)) .
$$

La convexité de $\Theta$ implique que, au point $s(y)$ la fonction $\psi$ vaut

$$
J(y)=\psi(s(y))=s(y) \cdot y-\Theta(y)+\Theta(-1,0) .
$$

Cela entraîne en particulier la relation

$$
\begin{gathered}
\partial_{t} J(\boldsymbol{y}(t))=\left(\partial_{t} \boldsymbol{s}(\boldsymbol{y}(t))\right) \cdot \boldsymbol{y}(t) \\
J(-1,0)=\mathbf{0}, \quad J(\boldsymbol{y}) \geq \mathbf{0}, \quad \forall \boldsymbol{y} \in \mathbb{R}^{2} .
\end{gathered}
$$

2) En pratique, les données d'un modèle d'écoulement triphasique, telles que perméabilités relatives et saturations, reposent sur des données expérimentales des modèles eau-huile et huile-gaz ( $c f .[2],[7])$.

Alors on peut considérer par exemple

$$
\begin{aligned}
K_{w} & =K_{w}\left(s_{w}\right), \quad s_{w}=s_{w}\left(p_{c w}\right) \\
K_{g} & =K_{g}\left(s_{g}\right), \quad s_{g}=s_{g}\left(p_{c g}\right),
\end{aligned}
$$


et on se réfere au modèle de Stone ou de Corey pour la dépendance de $K_{o}$ en fonction de $s_{w}$ et $s_{g}$.

Pour de telles saturations les conditions iv) et $\mathrm{v}$ ) de l'hypothèse (H1) sont évidemment vérifiées et l'existence de la fonction $\Theta$ définie en 1) est assurée.

Malheureusement des mesures récentes montrent l'insuffisance de tels modèles; par ailleurs lorsque la perméabilité relative d'une phase ne dépend que d'une seule saturation l'hypothèse de différentiabilité totale [7] n'est pas vérifiée. Dans ce cas l'existence d'une solution, même pour le problème régularisé, reste une question ouverte.

Par la suite, nous supposons que cette condition est satisfaite ainsi que l'hypothèse (H1).

En vue de simplifier l'écriture des équations, on introduit les notations suivantes :

$$
\begin{aligned}
& M_{\eta}\left(\boldsymbol{p}_{c}\right)=M_{\eta}\left(\boldsymbol{s}\left(\boldsymbol{p}_{c}\right)\right)=\frac{K_{\eta}}{\mu_{\eta}}: \text { mobilité de la phase } \eta, \eta=w, o, g, \\
& M\left(\boldsymbol{p}_{c}\right)=M_{w}+M_{o}+M_{g}: \text { mobilité totale } \\
& v_{\eta}\left(\boldsymbol{p}_{c}\right)=v_{\eta}\left(\boldsymbol{s}\left(\boldsymbol{p}_{c}\right)\right)=\frac{M_{\eta}}{M}: \text { fonction de flux de la phase } \eta .
\end{aligned}
$$

On note enfin $\pi$ l'ensemble des saturations admissibles défini par

$$
\Pi=\left\{\left(s_{w}, s_{g}\right) \in L^{\infty}(\Omega) \times L^{\infty}(\Omega), 0 \leq s_{w}, 0 \leq s_{g}, s_{w}+s_{g} \leq 1\right\} .
$$

Les fonctions $M$ et $v_{\eta}$ vérifient alors

$$
\left\{\begin{array}{l}
\exists m>0, M\left(s_{w}, s_{g}\right) \geq m, \forall\left(s_{w}, s_{g}\right) \in \mathbb{T} \\
v_{\eta}=v_{\eta}\left(s_{w}, s_{g}\right) \geq 0, \quad v_{o}+v_{g}+v_{w}=1 \\
v_{\eta} \text { s'annule lorsque } s_{\eta} \text { est nul, et } \\
v_{\eta} \text { vaut } 1 \text { lorsque } s_{\eta} \text { vaut } 1, \eta=w, o, g .
\end{array}\right.
$$

Dans [6]-[7], G. Chavent introduit une sous-classe de perméabilités relatives vérifiant une condition dite de "différentielle totale". Ceci permet de réduire le problème et d'en simplifier la formulation.

La condition de différentielle tolale de G. Chavent s'écrit :

$$
\frac{\partial v_{w}\left(\boldsymbol{p}_{c}\right)}{\partial p_{c g}}=\frac{\partial v_{g}\left(\boldsymbol{p}_{c}\right)}{\partial p_{c w}} \text {. }
$$

Cette hypothèse permet de définir une pression capillaire globale $\tilde{p}$ par

$$
\boldsymbol{\nabla} \tilde{p}\left(\boldsymbol{p}_{c}\right)=v_{w} \boldsymbol{\nabla} p_{c w}+v_{g} \boldsymbol{\nabla} p_{c g} .
$$


On trouve dans [8] un algorithme détaillé pour la détermination des perméabilités relatives triphasiques satisfaisant (H3). Ces perméabilités sont par ailleurs admissibles du point de vue de la mécanique des écoulements triphasiques.

\section{Formulation du problème}

Avant d'introduire les équations que doivent vérifier les inconnues du modèle, à savoir $p_{c w}, p_{c g}$ et $V_{T}=V_{w}+V_{o}+V_{g}$, nous allons préciser quelques notations :

$$
\begin{aligned}
& \bar{\rho}\left(\boldsymbol{p}_{c}\right)=\frac{M_{w} \rho_{w}+M_{o} \rho_{o}+M_{g} \rho_{g}}{M} \\
& \bar{M}_{w}\left(\boldsymbol{p}_{c}\right)=M v_{g} v_{o}, \quad \bar{M}_{g}=M v_{w} v_{o}, \quad \bar{M}_{o}=M v_{w} v_{g} \\
& \bar{\rho}_{w}\left(\boldsymbol{p}_{c}\right)=\bar{M}_{g}\left(\rho_{w}-\rho_{o}\right)+\bar{M}_{o}\left(\rho_{w}-\rho_{g}\right) \\
& \bar{\rho}_{g}\left(\boldsymbol{p}_{c}\right)=\bar{M}_{w}\left(\rho_{g}-\rho_{o}\right)+\bar{M}_{o}\left(\rho_{g}-\rho_{w}\right) .
\end{aligned}
$$

Équation en pression

En sommant les équations du système (2.1) et en tenant compte de (2.2) on obtient

$$
\operatorname{div} V_{T}=0
$$

avec

$$
V_{T}=V_{w}+V_{o}+V_{g}=-\mathbf{K}(x) M\left(\nabla_{p_{o}}+v_{w} \boldsymbol{\nabla} p_{c w}+v_{g} \boldsymbol{\nabla} p_{c g}-\bar{\rho} \boldsymbol{g}\right)
$$

En utilisant l'hypothèse (H3), on exprime la vitesse totale en fonction de la pression globale définie ci-dessous et de la gravité. L'équation (3.2) s'écrit alors

$$
V_{T}=-\mathbf{K}(x) M(\nabla p-\bar{\rho} \boldsymbol{g}),
$$

où $p$ est la pression globale définie par

$$
p=p_{o}+\tilde{p}
$$


Équations en pressions capillaires

On exprime tout d'abord $V_{w}$ et $V_{g}$ en fonction de $V_{T}$, d'où

$$
\begin{aligned}
& V_{w}=v_{w} V_{T}-\mathbf{K}(x)\left(\bar{M}_{g} \nabla_{p_{c w}}-\bar{M}_{o}\left(\nabla p_{c g}-\nabla p_{c w}\right)-\bar{\rho}_{w} \boldsymbol{g}\right) \\
& V_{g}=v_{g} V_{T}-\mathbf{K}(x)\left(\bar{M}_{w} \nabla_{p_{c g}}-\bar{M}_{o}\left(\nabla p_{c w}-\nabla p_{c g}\right)-\bar{\rho}_{g} \boldsymbol{g}\right) .
\end{aligned}
$$

Remplaçons les expressions de $V_{g}$ et $V_{w}$ données par (3.5) et (3.6) dans (2.1), on obtient

$$
\begin{aligned}
& \partial_{t}\left(\boldsymbol{\Phi}(x) s_{w}\left(\boldsymbol{p}_{c}\right)\right)+ \\
& \quad+\operatorname{div}\left(v_{w} V_{T}-\mathbf{K}(x)\left(\bar{M}_{g} \nabla p_{c w}-\bar{M}_{o}\left(\nabla p_{c g}-\nabla p_{c w}\right)-\bar{\rho}_{w} \boldsymbol{g}\right)\right)=0 \\
& \partial_{t}\left(\boldsymbol{\Phi}(x) s_{g}\left(\boldsymbol{p}_{c}\right)\right)+ \\
& \quad+\operatorname{div}\left(v_{g} V_{T}-\mathbf{K}(x)\left(\bar{M}_{w} \nabla p_{c g}-\bar{M}_{o}\left(\nabla p_{c w}-\nabla p_{c g}\right)-\bar{\rho}_{g} g\right)\right)=0 .
\end{aligned}
$$

Le système (3.1), (3.3), (3.7), (3.8) est de type parabolique-elliptique non linéaire dégénéré. En effet, les équations (3.7) et (3.8) présentent des termes de diffusion dégénérés car $v_{\eta}$ s'annule pour $s_{\eta}=0, \eta=w, o, g$.

Nous allons maintenant établir un résultat d'existence pour le système (3.1), (3.3), (3.7), (3.8) avec les conditions aux limites ci-dessous.

\section{Conditions aux limites}

On distingue, selon la nature des frontières considérées, les conditions aux limites suivantes :

- $\operatorname{sur} \Gamma_{e}$, partie de la frontière par laquelle l'eau est injectée on impose

$$
\left\{\begin{array}{l}
V_{T} \cdot \boldsymbol{n}=V_{w} \cdot \boldsymbol{n}=-q_{e}, \quad V_{g} \cdot \boldsymbol{n}=V_{o} \cdot n=0, \quad q_{e}>0 \\
p_{c w}=0\left(\text { i.e. } s_{w}=1\right), \quad p_{c g}=0\left(\text { i.e. } s_{g}=0\right) ;
\end{array}\right.
$$

- $\operatorname{sur} \Gamma_{i}$, frontière imperméable, on impose

$$
V_{T} \cdot \boldsymbol{n}=V_{w} \cdot n=V_{g} \cdot n=V_{o} \cdot n=0 ;
$$

- $\operatorname{sur} \Gamma_{s}$, frontière de récupération de l'huile et du gaz. 
On considère d'une part que le débit total des fluides récupérés $q_{s}$ à travers $\Gamma_{s}$ est strictement positif et, d'autre part, de façon analogue à [12] on considère que l'écoulement se produit sans flux de pression capillaire. Plus précisément les conditions aux limites s'écrivent

$$
\left\{\begin{array}{l}
V_{T} \cdot \boldsymbol{n}=q_{s} \\
\left(v_{o}+v_{g}\right) v_{w} \boldsymbol{\nabla} p_{c w} \cdot \boldsymbol{n}-v_{w} v_{g} \boldsymbol{\nabla} p_{c g} \cdot \boldsymbol{n}=\mathbf{0} \\
\left(v_{o}+v_{w}\right) v_{g} \boldsymbol{\nabla} p_{c g} \cdot \boldsymbol{n}-v_{g} v_{w} \boldsymbol{\nabla} p_{c w} \cdot \boldsymbol{n}=\mathbf{0} .
\end{array}\right.
$$

Le débit d'eau injectée à travers $\Gamma_{e}$ est lié au débit des fluides récupérés à travers $\Gamma_{s}$ par une condition de compatibilité qui traduit la continuité des phases dans le milieu ainsi que la condition d"incompressibilité

$$
\int_{\Gamma_{e}} q_{e} \mathrm{~d} \gamma_{e}-\int_{\Gamma_{s}} q_{s} \mathrm{~d} \gamma_{s}=0
$$

\section{Conditions initiales}

A l'instant initial $t=0, p_{c w}^{0}$ et $p_{c g}^{0}$ sont données par les relations

$$
\begin{cases}s_{w}^{0}(x)=s_{w}(0, x)=s_{w}\left(p_{c w}^{0}, p_{c g}^{0}\right) & \text { avec } 0 \leq s_{w}^{0} \leq 1 \text { p.p. dans } \Omega \\ s_{g}^{0}(x)=s_{g}(0, x)=s_{g}\left(p_{c w}^{0}, p_{c g}^{0}\right) & \text { avec } 0 \leq s_{g}^{0} \leq 1 \text { p.p. dans } \Omega \\ s_{w}^{0}+s_{g}^{0} \leq 1 . & \end{cases}
$$

Précisons maintenant quelques propriétés essentielles sur les données :

(H4) $q_{j} \in L^{\infty}\left(\Sigma_{j}\right), q_{j} \geq 0$ p.p. sur $\Sigma_{j}, j=e, s$;

(H5) $M$ et $v_{\eta}(\eta=w, o, g)$ sont des fonctions continues et bornées pour tout $\left(s_{w}, s_{g}\right)$ admissible.

$$
\exists \bar{m}_{\eta} \in \mathbb{R}_{+}, \eta=w, o, g \text { tel que } \bar{M}_{\eta}\left(\boldsymbol{p}_{c}\right) \geq \bar{m}_{\eta} ;
$$

(H6) $\mathbf{K}(x)=\left(k_{i j}(x)\right)_{i, j}, k_{i j}(x) \in L^{\infty}(\Omega)$, et il existe un réel $k$ strictement positif tel que :

$$
\sum_{i, j} k_{i j}(x) \xi_{i} \xi_{j} \geq k|\xi|^{2}, \quad \forall \xi \in \mathbb{R}^{n}, \text { p.p. } x \in \Omega ;
$$

(H7) $\Phi \in C^{1}(\Omega)$ et il existe des constantes $\varphi_{1}$ et $\varphi_{2}$ appartenant à $] 0,1[$ telles que :

$$
\varphi_{1} \leq \Phi(x) \leq \varphi_{2} \quad \text { p.p. dans } \Omega ;
$$

(Cette hypothèse est semblable à celle prise par Alt et Di Benedetto [1] pour des écoulements triphasiques en milieu poreux homogène.)

(H8) $\left(s_{w}^{0}, s_{g}^{0}\right) \in \pi$ où

$$
\Pi=\left\{\left(s_{w}, s_{g}\right) \in L^{\infty}(\Omega) \times L^{\infty}(\Omega), 0 \leq s_{\omega}, 0 \leq s_{g}, s_{w}+s_{g} \leq 1\right\} .
$$




\section{Cadre fonctionnel et formulation variationnelle}

On introduit les ensembles suivants :

$$
\begin{aligned}
& H^{1}(\Omega)=\left\{u \in L^{2}(\Omega) \mid \nabla u \in\left(L^{2}(\Omega)\right)^{3}\right\} \\
& \mathcal{V}=\left\{u \in H^{1}(\Omega) \mid u=0 \operatorname{sur} \Gamma_{e}\right\} \\
& \mathbb{H}=\left\{\boldsymbol{U} \in\left(L^{2}(\Omega)\right)^{3}|\operatorname{div} \boldsymbol{U}=\mathbf{0}, \boldsymbol{U} \cdot \boldsymbol{n}|_{\Gamma_{e}}=-q_{e},\left.\boldsymbol{U} \cdot \boldsymbol{n}\right|_{\Gamma_{i}}=\mathbf{0},\right. \\
& \left.\left.\boldsymbol{U} \cdot \boldsymbol{n}\right|_{\Gamma_{s}}=q_{s}\right\} .
\end{aligned}
$$

DÉFINITION 1. - Sous les hypothèses (H1)-(H8) on appelle solution faible du problème (3.1), (3.3), (3.7)-(3.13), noté (P), un quadruplet $\left(p_{c w}, p_{c g}, V_{T}, p\right)$ défini par : pour tout $t$ positif et $\left(s_{w}^{0}, s_{g}^{0}\right) \in \mathbb{\pi}$, alors :

$$
\left(s_{w}, s_{g}\right) \in \mathbb{\pi}, \quad \partial_{t}\left(\mathbf{\Phi}(x) s_{\eta}\left(\boldsymbol{p}_{c}\right)\right) \in L^{2}\left(\left[0, t\left[\times \mathcal{V}^{\prime}\right) \quad \text { où } \eta=w, g\right.\right.
$$

et

$$
\begin{aligned}
& \left(p_{c w}, p_{c g}\right) \in\left(L ^ { \infty } \left(\left[0, t[\times \Omega) \cap L^{2}\left([0, t[\times \mathcal{V}))^{2}\right.\right.\right.\right. \\
& V_{T} \in L^{\infty}\left(\left[0, t[, \mathbb{H}), \quad p \in L^{\infty}\left(\left[0, t\left[, H^{1}(\Omega) / \mathbb{R}\right)\right.\right.\right.\right.
\end{aligned}
$$

vérifiant $\forall u, v \in\left(L^{2}\left([0, t[\times \mathcal{V}))^{2}\right.\right.$ :

$$
\begin{aligned}
\int_{0}^{t}\left\langle\partial_{t}(\boldsymbol{\Phi}(x)\right. & \left.\left.s_{w}\left(\boldsymbol{p}_{c}\right)\right), u\right\rangle \mathrm{d} \tau-\int_{0}^{t} \int_{\Omega} \nu_{w}\left(\boldsymbol{p}_{c}\right) \boldsymbol{V}_{T} \cdot \nabla u \mathrm{~d} \omega \mathrm{d} \tau+ \\
& +\int_{0}^{t} \int_{\Omega} \mathbf{K}(x) \bar{M}_{g}\left(\boldsymbol{p}_{c}\right) \nabla p_{c w} \cdot \nabla u \mathrm{~d} \omega \mathrm{d} \tau+ \\
& +\int_{0}^{t} \int_{\Omega} \mathbf{K}(x) \bar{M}_{o}\left(\boldsymbol{p}_{c}\right)\left(\boldsymbol{\nabla} p_{c w}-\nabla p_{c g}\right) \cdot \nabla u \mathrm{~d} \omega \mathrm{d} \tau+ \\
& \quad-\int_{0}^{t} \int_{\Omega} \mathbf{K}(x) \bar{\rho}_{w}\left(\boldsymbol{p}_{c}\right) \boldsymbol{g} \cdot \boldsymbol{\nabla} u \mathrm{~d} \omega \mathrm{d} \tau= \\
= & -\int_{0}^{t} \int_{\Gamma_{s}} \nu_{w} q_{s} u \mathrm{~d} \gamma_{s} \mathrm{~d} \tau
\end{aligned}
$$


Existence de solutions faibles pour un modèle d'écoulement triphasique

$$
\begin{aligned}
\int_{0}^{t}\left\langle\partial_{t}(\boldsymbol{\Phi}(x)\right. & \left.\left.s_{g}\left(\boldsymbol{p}_{c}\right)\right), v\right\rangle \mathrm{d} \tau-\int_{0}^{t} \int_{\Omega} \nu_{g}\left(\boldsymbol{p}_{c}\right) V_{T} \cdot \nabla v \mathrm{~d} \boldsymbol{\omega} \tau+ \\
& +\int_{0}^{t} \int_{\Omega} \mathbf{K}(x) \bar{M}_{w}\left(\boldsymbol{p}_{c}\right) \nabla p_{c g} \cdot \nabla v \mathrm{~d} \boldsymbol{\omega} \mathrm{d} \tau+ \\
& +\int_{0}^{t} \int_{\Omega} \mathbf{K}(x) \bar{M}_{o}\left(\boldsymbol{p}_{c}\right)\left(\nabla p_{c g}-\nabla p_{c w}\right) \cdot \nabla v \mathrm{~d} \boldsymbol{d} \tau+ \\
& -\int_{0}^{t} \int_{\Omega} \mathbf{K}(x) \bar{\rho}_{g}\left(\boldsymbol{p}_{c}\right) \boldsymbol{g} \cdot \boldsymbol{\nabla} v \mathrm{~d} \boldsymbol{\omega} \mathrm{d} \tau= \\
= & -\int_{0}^{t} \int_{\Gamma_{s}} \nu_{g} q_{s} v \mathrm{~d} \gamma_{s} \mathrm{~d} \tau
\end{aligned}
$$

et $\forall w \in H^{1}(\Omega) / \mathbb{R}$ :

$$
\begin{gathered}
\int_{\Omega} \mathbf{K}(x) M\left(\boldsymbol{p}_{c}\right) \nabla p \cdot \nabla w \mathrm{~d} \omega-\int_{\Omega} \mathbf{K}(x) \bar{\rho}\left(\boldsymbol{p}_{c}\right) \boldsymbol{g} \cdot \nabla w \mathrm{~d} \omega= \\
=\int_{\Gamma_{e}} q_{e} w \mathrm{~d} \gamma_{e}-\int_{\Gamma_{\boldsymbol{s}}} q_{s} w \mathrm{~d} \gamma_{\boldsymbol{s}} .
\end{gathered}
$$

$V_{T}$ est donnée par

$$
V_{T}=-\boldsymbol{K}(x) M\left(\boldsymbol{p}_{c}\right)(\nabla p-\bar{\rho} \boldsymbol{g})
$$

5. Existence de solutions faibles pour le problème régularisé

Comme déjà vu les équations en pressions capillaires (3.7) et (3.8) sont de type parabolique dégénéré.

Pour régularisé l'opérateur on utilise la méthode de viscosité artificielle. On définit ainsi un problème régularisé $\left(\mathrm{P}_{\varepsilon}\right)$ avec $\varepsilon$ réel strictement positif, comme suit :

- trouver

$$
\begin{aligned}
& \boldsymbol{p}_{c}^{\varepsilon} \in\left(L ^ { \infty } \left(\left[0, t[\times \Omega) \cap L^{2}\left([0, t[\times \mathcal{V}))^{2}\right.\right.\right.\right. \\
& p^{\varepsilon} \in L^{\infty}\left(\left[0, t\left[, H^{1}(\Omega) / \mathbb{R}\right)\right.\right. \\
& \left(s_{w}^{\varepsilon}, s_{g}^{\varepsilon}\right) \in \mathbb{\pi}
\end{aligned}
$$


- vérifiant $\forall(u, v) \in\left(L^{2}\left([0, t[\times \mathcal{V}))^{2}\right.\right.$ :

$$
\begin{aligned}
\int_{0}^{t}\left\langle\partial_{t}\left(\boldsymbol{\Phi}(x) \boldsymbol{s}_{w}\left(\boldsymbol{p}_{c}^{\varepsilon}\right)\right), u\right\rangle \mathrm{d} \tau-\int_{0}^{t} \int_{\Omega} \nu_{w}\left(\boldsymbol{p}_{c}^{\varepsilon}\right) \boldsymbol{V}_{T}^{\varepsilon} \cdot \nabla u \mathrm{~d} \omega \mathrm{d} \tau+ \\
\quad+\int_{0}^{t} \int_{\Omega} \mathbf{K}(x) \bar{M}_{g}\left(p_{c}^{\varepsilon}\right) \nabla p_{c w}^{\varepsilon} \cdot \nabla u \mathrm{~d} \omega \mathrm{d} \tau+ \\
\quad+\int_{0}^{t} \int_{\Omega} \mathbf{K}(x) \bar{M}_{o}\left(\boldsymbol{p}_{c}^{\varepsilon}\right)\left(\nabla p_{c w}^{\varepsilon}-\nabla p_{c g}^{\varepsilon}\right) \cdot \nabla u \mathrm{~d} \omega \mathrm{d} \tau+ \\
\quad-\int_{0}^{t} \int_{\Omega} \mathbf{K}(x) \bar{\rho}_{w}\left(p_{c}^{\varepsilon}\right) \boldsymbol{g} \cdot \boldsymbol{\nabla} u \mathrm{~d} \omega \mathrm{d} \tau+ \\
\quad+\varepsilon \int_{0}^{t} \int_{\Omega} \nabla s_{w}\left(\boldsymbol{p}_{c}^{\varepsilon}\right) \cdot \nabla u \mathrm{~d} \omega \mathrm{d} \tau= \\
=-\int_{0}^{t} \int_{\Gamma_{s}} \nu_{w}\left(\boldsymbol{p}_{c}^{\varepsilon}\right) q_{s} u \mathrm{~d} \gamma_{s} \mathrm{~d} \tau
\end{aligned}
$$

$$
\begin{aligned}
\int_{0}^{t}\left\langle\partial_{t}\left(\boldsymbol{\Phi}(x) s_{g}\left(\boldsymbol{p}_{c}^{\varepsilon}\right)\right), v\right\rangle \mathrm{d} \tau-\int_{0}^{t} \int_{\Omega} \nu_{g}\left(\boldsymbol{p}_{c}^{\varepsilon}\right) \boldsymbol{V}_{T}^{\varepsilon} \cdot \nabla v \mathrm{~d} \omega \mathrm{d} \tau+ \\
\quad+\int_{0}^{t} \int_{\Omega} \mathbf{K}(x) \bar{M}_{w}\left(\boldsymbol{p}_{c}^{\varepsilon}\right) \nabla p_{c g}^{\varepsilon} \cdot \nabla v \mathrm{~d} \omega \mathrm{d} \tau+ \\
\quad+\int_{0}^{t} \int_{\Omega} \boldsymbol{K}(x) \bar{M}_{o}\left(\boldsymbol{p}_{c}^{\varepsilon}\right)\left(\nabla p_{c g}^{\varepsilon}-\nabla p_{c w}^{\varepsilon}\right) \cdot \nabla v \mathrm{~d} \omega \mathrm{d} \tau+ \\
\quad-\int_{0}^{t} \int_{\Omega} \mathbf{K}(x) \bar{\rho}_{g}\left(\boldsymbol{p}_{c}^{\varepsilon}\right) \boldsymbol{g} \cdot \nabla v \mathrm{~d} \omega \mathrm{d} \tau+ \\
\quad+\varepsilon \int_{0}^{t} \int_{\Omega} \boldsymbol{\nabla} s_{g}\left(\boldsymbol{p}_{c}^{\varepsilon}\right) \cdot \nabla v \mathrm{~d} \omega \mathrm{d} \tau= \\
=-\int_{0}^{t} \int_{\Gamma_{s}} \nu_{g}\left(\boldsymbol{p}_{c}^{\varepsilon}\right) q_{s} v \mathrm{~d} \gamma_{s} \mathrm{~d} \tau .
\end{aligned}
$$

$$
\begin{array}{r}
\int_{\Omega} \mathbf{K}(x) M\left(p_{c}^{\varepsilon}\right) \nabla p^{\varepsilon} \cdot \nabla w \mathrm{~d} \omega-\int_{\Omega} \mathbf{K}(x) \bar{\rho}\left(\boldsymbol{p}_{c}^{\varepsilon}\right) \boldsymbol{g} \cdot \nabla w \mathrm{~d} \omega= \\
=\int_{\Gamma_{s}} q_{e} w \mathrm{~d} \gamma_{e}-\int_{\Gamma_{s}} q_{s} w \mathrm{~d} \gamma_{s}, \quad \forall w \in H^{1}(\Omega) / \mathbb{R}
\end{array}
$$

$V_{T}^{\varepsilon}$ est donnée par

$$
V_{T}^{\varepsilon}=-\mathbf{K}(x) M\left(\boldsymbol{p}_{c}^{\varepsilon}\right)\left(\nabla p^{\varepsilon}-\bar{\rho} \boldsymbol{g}\right) .
$$


L'étude du problème dégénéré sera traitée dans le paragraphe 6 .

THÉORÈME 1. - Sous les hypothèses (H1)-(H8), pour tout $\varepsilon$ strictement positif, le problème régularisé $\left(P_{\varepsilon}\right)$ admet au moins une solution faible.

La démonstration de ce théorème se fait en plusieurs étapes.

Étape 1 : Approximation des solutions pour le problème régularisé

La méthode utilisée afin d'approcher les solutions du problème $\left(P_{\epsilon}\right)$ consiste à découpler le système en introduisant un retard en temps.

Un tel schéma revient à prendre pour la mobilité de chaque phase la valeur prise à l'intervalle de temps précédent. Ce schéma est semblable à celui pris par P. Fabrie et M. Langlais dans [10].

Dans [3], [12] et [13], des schémas semi-implicites pour des problèmes semi-discrétisés en temps, modélisant soit un écoulement triphasique compressible soit un écoulement diphasique incompressible en milieu poreux hétérogène, sont proposés. Pour ces schémas, les auteurs précédents établissent une estimation $L^{\infty}$ à chaque étape; le passage à la limite n'est pas envisagé dans ces études.

Nous allons prouver ici que l'algorithme (A) ci-dessous est convergent. La première étape consiste à prolonger de façon convenable les mobilités et les saturations sur $\mathbb{R} \times \mathbb{R}$ puisqu'on ne sait pas, a priori, que les saturations appartiennent au convexe admissible $\pi$.

(H9) Hypothèse de prolongement

On prolonge les fonctions $\nu_{w}, \nu_{g}, \nu_{o}$ et $M$ en des fonctions $\tilde{\nu}_{w}, \tilde{\nu}_{g}, \tilde{\nu}_{o}$ et $\widetilde{M}$ continues et bornées sur $\mathbb{R} \times \mathbb{R}$. Un tel prolongement est explicité dans l'étape 3, on en admet l'existence pour les étapes 1 et 2 .

L'hypothèse $(\mathrm{H} 1)$ permet ensuite de prolonger par troncature et régularisation les fonctions $\boldsymbol{s}_{w}\left(\boldsymbol{p}_{c}\right)$ et $\boldsymbol{s}_{g}\left(\boldsymbol{p}_{c}\right)$ en des fonctions $\tilde{\boldsymbol{s}}_{w}\left(\boldsymbol{p}_{c}\right)$ et $\tilde{\boldsymbol{s}}_{g}\left(\boldsymbol{p}_{c}\right)$ de classe $C^{1}\left(\mathbf{R}^{2}, \mathbb{R}\right)$ à dérivées bornées telles que

$$
\begin{gathered}
\boldsymbol{s}_{\eta}^{1} \leq \frac{\partial \tilde{s}_{\eta}}{\partial p_{c \eta}}(\boldsymbol{y}) \leq \boldsymbol{s}_{\eta}^{2}, \quad \forall \boldsymbol{y} \in \mathbb{R}^{2}, \eta=w, g \\
\boldsymbol{s}_{\eta}^{3} \leq \frac{\partial \tilde{s}_{\eta}}{\partial p_{c \mu}}(\boldsymbol{y}) \leq \boldsymbol{s}_{\eta}^{4}, \quad \forall \boldsymbol{y} \in \mathbb{R}^{2},(\eta, \mu)=(w, g),(g, w),
\end{gathered}
$$

avec

$$
\begin{cases}s_{\eta}^{i} \text { réel strictement positif } & i=1,2 \\ s_{\eta}^{i} \text { réel strictement négatif } & i=3,4\end{cases}
$$


Soit $h$ un nombre réel positif. Pour toute fonction $f: \mathbb{R} \times \Omega \rightarrow \mathbb{R}$, on note

$$
\tau_{h} f(t, x)=f(t-h, x) .
$$

On définit alors l'algorithme (A) ci-dessous :

Algorithme (A). - On pose

$$
p_{c w}^{h}(t, x)=p_{c w}^{0}(x) \quad \text { et } \quad p_{c g}^{h}(t, x)=p_{c g}^{0}(x) \quad \text { pour } t \in[-h, 0], x \in \Omega .
$$

On suppose que $p_{c w}^{h}(t, x)$ et $p_{c g}^{h}(t, x)$ sont données $\operatorname{sur}[k h,(k+1) h[$; on détermine alors $p^{h}$ vérifiant

$$
p^{h} \in L^{\infty}\left(\left[k h,(k+1) h\left[; H^{1}(\Omega) / \mathbb{R}\right)\right.\right.
$$

telle que $\forall \psi \in H^{1}(\Omega) / \mathbb{R}$,

$$
\begin{aligned}
& \int_{\Omega} \mathbf{K}(x) \widetilde{M}\left(p_{c}^{h}\right) \boldsymbol{\nabla} p^{h} \cdot \nabla \psi \mathrm{d} \omega= \\
& \quad=\int_{\Omega} \mathbf{K}(x) \tilde{\bar{\rho}}\left(\boldsymbol{p}_{c}^{h}\right) \boldsymbol{g} \cdot \boldsymbol{\nabla} \psi \mathrm{d} \omega+\int_{\Gamma_{e}} q_{e} \psi \mathrm{d} \gamma_{e}-\int_{\Gamma_{\boldsymbol{s}}} q_{\boldsymbol{s}} \psi \mathrm{d} \gamma_{\boldsymbol{s}} .
\end{aligned}
$$

La vitesse totale $V_{T}^{h}$ est alors donnée par

$$
\begin{gathered}
\boldsymbol{V}_{T}^{h} \in L^{\infty}([k h,(k+1) h[, \mathbb{H}) \\
\boldsymbol{V}_{T}^{h}=-\mathbf{K}(x) \widetilde{M}\left(\boldsymbol{p}_{c}^{h}\right)\left(\boldsymbol{\nabla} p^{h}-\tilde{\bar{\rho}}\left(\boldsymbol{p}_{c}^{h}\right) \boldsymbol{g}\right) .
\end{gathered}
$$

On définit ensuite $p_{c w}^{h}$ et $p_{c g}^{h}$ sur l'intervalle $[(k+1) h,(k+2) h$ [ vérifiant

$$
\begin{aligned}
\left(p_{c w}^{h}, p_{c g}^{h}\right) \in\left(L ^ { \infty } \left(\left[(k+1) h,(k+2) h\left[, L^{2}(\Omega)\right)\right.\right.\right. & \cap \\
& \cap L^{2}\left([(k+1) h,(k+2) h[, \mathcal{V}))^{2}\right.
\end{aligned}
$$

et telles que $\forall(u, v) \in\left(L_{\text {loc }}^{2}\left(\mathbb{R}_{+}, \mathcal{V}\right)\right)^{2}$ :

$$
\begin{aligned}
\int_{(k+1) h}^{(k+2) h}\left\langle\partial_{t}\left(\Phi(x) \tilde{s}_{w}\left(\boldsymbol{p}_{c}^{h}\right)\right), u\right\rangle \mathrm{d} t-\int_{(k+1) h}^{(k+2) h} \int_{\Omega} \tau_{h}\left(\tilde{\nu}_{w}^{h}\right) V_{T}^{h} \cdot \nabla u \mathrm{~d} \omega \mathrm{d} t+ \\
+\int_{(k+1) h}^{(k+2) h} \int_{\Omega} \mathbf{K}(x) \tau_{h}\left(\widetilde{\bar{M}}_{g}^{h}\right) \nabla p_{c w}^{h} \cdot \nabla u \mathrm{~d} \omega \mathrm{d} t+
\end{aligned}
$$


Existence de solutions faibles pour un modèle d'écoulement triphasique

$$
\begin{aligned}
& +\int_{(k+1) h}^{(k+2) h} \int_{\Omega} \mathbf{K}(x) \tau_{h}\left(\widetilde{\bar{M}}_{o}^{h}\right) \nabla\left(p_{c w}^{h}-p_{c g}^{h}\right) \cdot \nabla u \mathrm{~d} \omega \mathrm{d} t+ \\
& -\int_{(k+1) h}^{(k+2) h} \int_{\Omega} \mathbf{K}(x) \tau_{h}\left(\tilde{\bar{\rho}}_{w}^{h}\right) \boldsymbol{g} \cdot \nabla u \mathrm{~d} \omega \mathrm{d} t+ \\
& +\varepsilon \int_{(k+1) h}^{(k+2) h} \int_{\Omega} \nabla \tilde{s}_{w}\left(p_{c}^{h}\right) \cdot \nabla u \mathrm{~d} \omega \mathrm{d} \tau= \\
& =-\int_{(k+1) h}^{(k+2) h} \int_{\Gamma_{s}} \tau_{h}\left(\widetilde{\nu}_{w}^{h}\right) q_{s} u \mathrm{~d} \gamma_{s} \mathrm{~d} t \\
& \int_{(k+1) h}^{(k+2) h}\left\langle\partial_{t}\left(\Phi(x) \tilde{s}_{g}\left(p_{c}^{h}\right)\right), v\right\rangle \mathrm{d} t-\int_{(k+1) h}^{(k+2) h} \int_{\Omega} \tau_{h}\left(\widetilde{\nu}_{g}^{h}\right) V_{T}^{h} \cdot \nabla v \mathrm{~d} \omega \mathrm{d} t+ \\
& +\int_{(k+1) h}^{(k+2) h} \int_{\Omega} \mathbf{K}(x) \tau_{h}\left(\widetilde{\bar{M}}_{w}^{h}\right) \nabla p_{c g}^{h} \cdot \nabla v \mathrm{~d} \omega \mathrm{d} t+ \\
& +\int_{(k+1) h}^{(k+2) h} \int_{\Omega} \mathbf{K}(x) \tau_{h}\left(\widetilde{\bar{M}}_{o}^{h}\right) \nabla\left(p_{c g}^{h}-p_{c w}^{h}\right) \cdot \nabla v \mathrm{~d} \omega \mathrm{d} t+ \\
& -\int_{(k+1) h}^{(k+2) h} \int_{\Omega} \mathbf{K}(x) \tau_{h}\left(\tilde{\bar{\rho}}_{g}^{h}\right) \boldsymbol{g} \cdot \nabla v \mathrm{~d} \boldsymbol{\omega} \mathrm{d} t+ \\
& +\varepsilon \int_{(k+1) h}^{(k+2) h} \int_{\Omega} \nabla \tilde{s}_{g}\left(p_{c}^{h}\right) \cdot \nabla v \mathrm{~d} \omega \mathrm{d} \tau= \\
& =-\int_{(k+1) h}^{(k+2) h} \int_{\Gamma_{s}} \tau_{h}\left(\widetilde{\nu}_{g}^{h}\right) q_{s} v \mathrm{~d} \gamma_{s} \mathrm{~d} t .
\end{aligned}
$$

Proposition 1.- Sous les hypothèses (H1)-(H9), pour tout $\varepsilon$ strictement positif, l'algorithme (A) est consistant et admet une solution $\left(p_{c w}^{h}, p_{c g}^{h}, p^{h}, V_{T}^{h}\right)$ satisfaisant

$$
\begin{aligned}
& \left(p_{c w}^{h}, p_{c g}^{h}\right) \in\left(L^{\infty}\left(\mathbb{R}_{+}, L^{2}(\Omega)\right) \cap L_{\text {loc }}^{2}\left(\mathbb{R}_{+}, \mathcal{V}\right)\right)^{2} \\
& V_{T}^{h} \in L^{\infty}\left(\mathbb{R}_{+}, \mathbb{H}\right), \quad p^{h} \in L^{\infty}\left(\mathbb{R}_{+}, H^{1}(\Omega) / \mathbb{R}\right) .
\end{aligned}
$$

De plus, il existe des constantes $k_{i}, i=1,4$, dépendant des conditions initiales, des données $q_{e}, q_{s}$ et indépendantes de $h$ et de $\varepsilon$ telles que

$$
\begin{gathered}
\varepsilon^{2} \int_{0}^{t}\left|\nabla p_{c g}^{h}\right|_{L^{2}(\Omega)}^{2} \mathrm{~d} \tau \leq k_{1}, \quad \varepsilon^{2} \int_{0}^{t}\left|\nabla p_{c g}^{h}\right|_{L^{2}(\Omega)}^{2} \mathrm{~d} \tau \leq k_{2} \\
\left|V_{T}^{h}\right|_{L^{\infty}\left(\mathbb{R}_{+}, \mathbb{H}\right)} \leq k_{3}, \quad\left|p^{h}\right|_{L^{\infty}\left(\mathbb{R}_{+}, H^{1}(\Omega) / \mathbb{R}\right)} \leq k_{4},
\end{gathered}
$$


et il existe des constantes $k_{5}, k_{6}$ dépendant de $k_{i}, i=1,4$ et de $\varepsilon$ telles que

$$
\begin{aligned}
& \left|\partial_{t}\left(\Phi(x) \tilde{s}_{w}\left(p_{c}^{h}\right)\right)\right|_{L^{2}\left(0, t, \mathcal{V}^{\prime}\right)} \leq k_{5}(\varepsilon), \\
& \left|\partial_{t}\left(\Phi(x) \tilde{s}_{g}\left(p_{c}^{h}\right)\right)\right|_{L^{2}\left(0, t, \mathcal{V}^{\prime}\right)} \leq k_{6}(\varepsilon) .
\end{aligned}
$$

Preuve de la proposition 1

L'existence d'une solution pour le système (5.8)-(5.11) peut s'obtenir par exemple par la méthode de Galerkin puis par passage à la limite à l'aide d'estimations a priori du type de celles ci-dessous. En effet, le système à résoudre peut s'écrire formellement :

$$
\frac{\mathrm{d}}{\mathrm{d} t} f(u)+A(t) u=g(t)
$$

où $A$ est un opérateur linéaire et $f$ une application de classe $C^{1}$ de jacobienne inversible. Ceci assure l'existence d'une solution locale pour les approximants de Galerkin, les estimations a priori obtenues montrant en fait que cette solution est globale.

Nous allons donc établir les estimations ci-dessous dans lesquelles on omet l'indice $h$.

\section{Estimation de l'équation de Darcy}

On rappelle tout d'abord que l'application $\dot{v} \rightarrow|\nabla v|_{L^{2}(\Omega)}$ est une norme équivalente à

$$
\|\dot{v}\|_{H^{1}(\Omega) / \mathbb{R}}=\inf _{v \in \dot{v}}\left(\|v\|_{H^{1}(\Omega)}\right) \quad(\text { voir [14, théorème 1.9]). }
$$

Le membre de droite de l'équation (5.8) est une forme linéaire et continue sur $H^{1}(\Omega)$. La condition de compatibilité (3.12) étant vérifiée, cette forme linéaire est donc définie et continue sur $H^{1}(\Omega) / \mathbb{R}$.

Des hypothèses $(\mathrm{H} 2),(\mathrm{H} 6)$ et du résultat d'équivalence des normes dans $H^{1}(\Omega) / \mathbb{R}$ rappelé ci-dessus, le membre de gauche de l'équation (5.8) est une forme bilinéaire continue et coercive sur $H^{1}(\Omega) / \mathbb{R}$. Donc d'après le théorème de Lax-Milgram, il existe un unique $p$ dans $H^{1}(\Omega) / \mathbb{R}$ solution de (5.8) si et seulement si la condition (3.12) est vérifiée.

En prenant $\psi=p$ dans l'équation (5.8), on obtient d'après (H3), (H5) et (H9) l'estimation suivante :

$$
\begin{aligned}
k m|\nabla p|_{L^{2}(\Omega)}^{2} \leq & \operatorname{mes}(\Omega)\left|\mathbf{K}(x) \tilde{\bar{\rho}}\left(p_{c}\right) \boldsymbol{g}\right|_{L^{\infty}(\Omega)}|\nabla p|_{L^{2}(\Omega)}+ \\
& +\left|q_{e}\right|_{L^{2}\left(\Gamma_{e}\right)}|p|_{L^{2}\left(\Gamma_{e}\right)}+\left|q_{s}\right|_{L^{2}\left(\Gamma_{e}\right)}|p|_{L^{2}\left(\Gamma_{e}\right)} . \\
& -352-
\end{aligned}
$$


Par continuité de l'application trace définie de $H^{1}(\Omega)$ dans $H^{1 / 2}(\Gamma)$ dont l'injection dans $L^{2}(\Gamma)$ est continue, on déduit de (5.12) l'estimation

$$
k m|\nabla p|_{L^{2}(\Omega)}^{2} \leq c \inf _{p \in p}\|p\|_{H^{1}(\Omega)}
$$

enfin dans $H^{1}(\Omega) / \mathbb{R}$, il existe une constante $c_{1}$ dépendant uniquement des données telles que

$$
|\nabla p|_{L^{2}(\Omega)}^{2} \leq c_{1}
$$

Par conséquent en utilisant l'estimation (5.13) et l'égalité (5.9), il existe une constante $c_{2}$ dépendant uniquement des données telles que :

$$
\left|V_{T}\right|_{L^{2}(\Omega)}^{2} \leq c_{2} .
$$

\section{Estimation sur les lois de conservations}

En prenant dans l'équation (5.10) $u=p_{c w}$ et dans l'équation (5.11) $v=p_{c g}$ et en sommant ces équations, on obtient

$$
\begin{aligned}
& \left\langle\partial_{t}\left(\boldsymbol{\Phi}(x) \tilde{\boldsymbol{s}}_{w}\left(\boldsymbol{p}_{c}\right)\right), p_{c w}\right\rangle+\left\langle\partial_{t}\left(\boldsymbol{\Phi}(x) \tilde{\boldsymbol{s}}_{g}\left(\boldsymbol{p}_{c}\right)\right), p_{c g}\right\rangle+ \\
& +\int_{\Omega} \mathbf{K}(x) \widetilde{\bar{M}}_{g} \nabla p_{c w} \cdot \nabla p_{c w} \mathrm{~d} \omega+\int_{\Omega} \mathbf{K}(x) \widetilde{\bar{M}}_{w} \nabla p_{c g} \cdot \nabla p_{c g} \mathrm{~d} \omega+ \\
& +\int_{\Omega} \mathbf{K}(x) \widetilde{\bar{M}}_{o}\left(\nabla p_{c w}-\nabla p_{c g}\right)\left(\nabla p_{c w}-\nabla p_{c g}\right) \mathrm{d} \omega+ \\
& +\varepsilon \int_{\Omega} \nabla \tilde{\boldsymbol{s}}_{w}\left(\boldsymbol{p}_{c}\right) \cdot \boldsymbol{\nabla} p_{c w} \mathrm{~d} \omega+\varepsilon \int_{\Omega} \boldsymbol{\nabla} \tilde{\boldsymbol{s}}_{g}\left(\boldsymbol{p}_{c}\right) \cdot \boldsymbol{\nabla} p_{c g} \mathrm{~d} \omega= \\
& =\int_{\Omega} \tilde{\nu}_{w} V_{T} \cdot \nabla p_{c w} \mathrm{~d} \omega+\int_{\Omega} \tilde{\nu}_{g} V_{T} \cdot \nabla p_{c g} \mathrm{~d} \omega+ \\
& -\int_{\Gamma_{s}} \tilde{\nu}_{w} q_{s} p_{c w} \mathrm{~d} \gamma_{s}-\int_{\Gamma_{s}} \tilde{\nu}_{g} q_{s} p_{c g} \mathrm{~d} \gamma_{s} .
\end{aligned}
$$

En utilisant la relation

$$
\boldsymbol{\nabla} \tilde{\boldsymbol{s}}_{\eta}\left(\boldsymbol{p}_{c}\right)=\frac{\partial \tilde{\boldsymbol{s}}_{\eta}}{\partial p_{c w}}\left(\boldsymbol{p}_{c}\right) \boldsymbol{\nabla} p_{c w}+\frac{\partial \tilde{\boldsymbol{s}}_{\eta}}{\partial p_{c g}}\left(\boldsymbol{p}_{c}\right) \nabla p_{c g}
$$


et d'après les hypothèses (H3), (H5), (H9), on obtient

$$
\begin{aligned}
& \left\langle\partial_{t}\left(\boldsymbol{\Phi}(x) \tilde{\boldsymbol{s}}_{w}\left(\boldsymbol{p}_{c}\right)\right), p_{c w}\right\rangle+\left\langle\partial_{t}\left(\boldsymbol{\Phi}(x) \tilde{\boldsymbol{s}}_{g}\left(\boldsymbol{p}_{c}\right)\right), p_{c g}\right\rangle+ \\
& +k \bar{m}_{g}\left|\nabla p_{c w}\right|_{L^{2}(\Omega)}^{2}+k \bar{m}_{w}\left|\nabla p_{c g}\right|_{L^{2}}^{2}+ \\
& +\varepsilon \int_{\Omega} \frac{\partial \tilde{s}_{w}}{\partial p_{c w}}\left(\boldsymbol{p}_{c}\right)\left|\nabla p_{c w}\right|^{2} \mathrm{~d} \omega+\varepsilon \int_{\Omega} \frac{\partial \tilde{\boldsymbol{s}}_{g}}{\partial p_{c g}}\left(\boldsymbol{p}_{c}\right)\left|\nabla p_{c g}\right|^{2} \mathrm{~d} \omega \leq \\
& \leq \varepsilon \int_{\Omega}\left|\frac{\partial \tilde{s}_{w}}{\partial p_{c g}}\left(\boldsymbol{p}_{c}\right) \nabla p_{c g} \cdot \nabla p_{c w}\right| \mathrm{d} \omega+ \\
& +\varepsilon \int_{\Omega}\left|\frac{\partial \tilde{s}_{g}}{\partial p_{c w}}\left(\boldsymbol{p}_{c}\right) \nabla p_{c g} \cdot \nabla p_{c w}\right| \mathrm{d} \omega+ \\
& +\left|\tilde{\nu}_{w}\right|_{L^{\infty}(\Omega)}\left|V_{T}\right|_{L^{2}(\Omega)}\left|\nabla p_{c w}\right|_{L^{2}(\Omega)}+ \\
& +\left|\tilde{\nu}_{g}\right|_{L^{\infty}(\Omega)}\left|V_{T}\right|_{L^{2}(\Omega)}\left|\nabla p_{c g}\right|_{L^{2}(\Omega)}+ \\
& +\left|\tilde{\nu}_{w}\right|_{L^{\infty}\left(\Gamma_{s}\right)}\left|q_{s}\right|_{L^{2}\left(\Gamma_{s}\right)}\left|p_{c w}\right|_{L^{2}\left(\Gamma_{s}\right)}+ \\
& +\left|\tilde{\nu}_{g}\right|_{L^{\infty}\left(\Gamma_{s}\right)}\left|q_{s}\right|_{L^{2}\left(\Gamma_{s}\right)}\left|p_{c g}\right|_{L^{2}\left(\Gamma_{s}\right)} .
\end{aligned}
$$

Le premier terme du membre de droite de l'inégalité précédente se majore grâce à (H9) et à l'inégalité de Young par

$$
\frac{\varepsilon}{2}\left|s_{w}^{3}\right|\left(\left|\nabla p_{c g}\right|_{L^{2}(\Omega)}^{2}+\left|\nabla p_{c w}\right|_{L^{2}(\Omega)}^{2}\right) ;
$$

de même, le deuxième terme du membre de droite se majore par

$$
\frac{\varepsilon}{2}\left|s_{g}^{3}\right|\left(\left|\nabla p_{c g}\right|_{L^{2}(\Omega)}^{2}+\left|\nabla p_{c w}\right|_{L^{2}(\Omega)}^{2}\right) .
$$

On note

$$
\begin{aligned}
& \beta_{1}(\varepsilon)=k \bar{m}_{g}+\varepsilon\left(s_{w}^{1}-\max \left(\left|s_{w}^{3}\right|,\left|s_{g}^{3}\right|\right)\right) \\
& \beta_{2}(\varepsilon)=k \bar{m}_{w}+\varepsilon\left(s_{g}^{1}-\max \left(\left|s_{w}^{3}\right|,\left|s_{g}^{3}\right|\right)\right) .
\end{aligned}
$$

D'après l'hypothèse $(\mathrm{H} 1)$

$$
s_{\eta}^{1}-\max \left(\left|s_{w}^{3}\right|,\left|s_{g}^{3}\right|\right)
$$

est strictement positif, pour $\eta=w, g$. Ainsi, $\beta_{1}(\varepsilon)$ et $\beta_{2}(\varepsilon)$ sont strictement positifs. 
On applique alors une fois de plus l'inégalité de Young, et compte tenu des majorations faites ci-dessus et de l'estimation (5.16), on obtient

$$
\begin{aligned}
& \left\langle\partial_{t}\left(\boldsymbol{\Phi}(x) \tilde{\boldsymbol{s}}_{w}\left(\boldsymbol{p}_{c}\right)\right), p_{c w}\right\rangle+\left\langle\partial_{t}\left(\boldsymbol{\Phi}(\boldsymbol{x}) \tilde{\boldsymbol{s}}_{g}\left(\boldsymbol{p}_{c}\right)\right), p_{c g}\right\rangle+ \\
& +\frac{\beta_{1}(\varepsilon)}{2}\left|\nabla p_{c w}\right|_{L^{2}(\Omega)}^{2}+\frac{\beta_{2}(\varepsilon)}{2}\left|\nabla p_{c g}\right|_{L^{2}(\Omega)}^{2} \leq \\
& \quad \leq \frac{c_{3}}{\beta_{1}(\varepsilon)}+\frac{c_{4}}{\beta_{2}(\varepsilon)} .
\end{aligned}
$$

Pour estimer les deux premiers termes de l'inégalité (5.17), on utilise la fonction $J$ introduite dans la remarque 1 , où $J$ vaut ici

$$
J\left(\boldsymbol{p}_{c}\right)=\tilde{\boldsymbol{s}}\left(\boldsymbol{p}_{c}\right) p_{c}-\Theta\left(\boldsymbol{p}_{c}\right)+\boldsymbol{\Theta}(-1,0) .
$$

Par suite, l'inégalité (5.17) est équivalente à l'inégalité suivante

$$
\begin{gathered}
\frac{\mathrm{d}}{\mathrm{d} t} \int_{\Omega} \Phi(x) J\left(p_{c}\right) \mathrm{d} \omega+\frac{\beta_{1}(\varepsilon)}{2}\left|\nabla p_{c w}\right|_{L^{2}(\Omega)}^{2}+\frac{\beta_{2}(\varepsilon)}{2}\left|\nabla p_{c g}\right|_{L^{2}(\Omega)}^{2} \leq \\
\leq \frac{c_{3}}{\beta_{1}(\varepsilon)}+\frac{c_{4}}{\beta_{2}(\varepsilon)} .
\end{gathered}
$$

En intégrant cette inégalité entre 0 et $t$, on a

$$
\begin{gathered}
\int_{\Omega} \Phi(x) J\left(p_{c}(t)\right) \mathrm{d} \omega+\frac{\beta_{1}(\varepsilon)}{2} \int_{0}^{t}\left|\nabla p_{c w}(\tau)\right|_{L^{2}(\Omega)}^{2} \mathrm{~d} \tau+ \\
+\frac{\beta_{2}(\varepsilon)}{2} \int_{0}^{t}\left|\nabla p_{c g}(\tau)\right|_{L^{2}(\Omega)}^{2} \mathrm{~d} \tau \leq \\
\leq \frac{c_{3} t}{\beta_{1}(\varepsilon)}+\frac{c_{4} t}{\beta_{2}(\varepsilon)}+\int_{\Omega} \Phi(x) J\left(p_{c}^{0}\right) \mathrm{d} \omega .
\end{gathered}
$$

Le troisième terme du membre de droite est une constante dépendant uniquement des conditions initiales. De plus, la positivité de la fonction $J$ permet d'obtenir de (5.19) les estimations

$$
\begin{gathered}
\int_{0}^{t}\left|\nabla p_{c w}(\tau)\right|_{L^{2}(\Omega)}^{2} \mathrm{~d} \tau \leq c_{5}(\varepsilon) \\
\int_{0}^{t}\left|\nabla p_{c g}(\tau)\right|_{L^{2}(\Omega)}^{2} \mathrm{~d} \tau \leq c_{6}(\varepsilon) \\
\sup _{\tau \in[0, t[} \int_{\Omega} J\left(p_{c}(\tau)\right) \mathrm{d} \omega \leq c_{7}(\varepsilon) .
\end{gathered}
$$


L'inégalité (5.19) précise le comportement des constantes $c_{5}$ et $c_{6}$ en fonction de $\varepsilon$. En effet,

$$
\int_{0}^{t}\left|\nabla p_{c w}(\tau)\right|_{L^{2}(\Omega)}^{2} \mathrm{~d} \tau \leq \frac{2 c_{3} t}{\beta_{1}(\varepsilon) \cdot \beta_{1}(\varepsilon)}+\frac{2 c_{4} t}{\beta_{2}(\varepsilon) \cdot \beta_{2}(\varepsilon)}+\frac{\int_{\Omega} \Phi(x) J\left(p_{c}^{0}\right) \mathrm{d} \omega}{\beta_{1}(\varepsilon)} .
$$

Or, $\beta_{1}(\varepsilon)$ et $\beta_{2}(\varepsilon)$ sont des fonctions linéaires en $\varepsilon$, par suite pour $\varepsilon$ assez petit on a

$$
\varepsilon^{2} \int_{0}^{t}\left|\nabla p_{c w}(\tau)\right|_{L^{2}(\Omega)}^{2} \mathrm{~d} \tau \leq c_{8}
$$

De même

$$
\varepsilon^{2} \int_{0}^{t}\left|\nabla p_{c g}(\tau)\right|_{L^{2}(\Omega)}^{2} \mathrm{~d} \tau \leq c_{9}
$$

Puisque les dérivées partielles des saturations $\tilde{s}_{w}$ et $\tilde{s}_{g}$ par rapport à $p_{c w}$ et $p_{c g}$ sont bornées, on déduit des estimations précédentes

$$
\begin{aligned}
& \varepsilon^{2} \int_{0}^{t} \int_{\Omega}\left|\nabla \tilde{s}_{w}\left(p_{c}\right)\right|^{2} \mathrm{~d} \omega \mathrm{d} \tau \leq c_{10} \\
& \varepsilon^{2} \int_{0}^{t} \int_{\Omega}\left|\nabla \tilde{s}_{g}\left(p_{c}\right)\right|^{2} \mathrm{~d} \omega \mathrm{d} \tau \leq c_{11} .
\end{aligned}
$$

Ces estimations sont essentielles pour la section 6 .

Nous allons établir une estimation $\left(L^{\infty}\left(\left[0, t\left[\times L^{2}(\Omega)\right)\right)^{2}\right.\right.$ sur $\boldsymbol{p}_{c}$ à partir de l'estimation (5.22). Pour cela, on écrit

$$
J\left(p_{c w}, p_{c g}\right)=J(-1,0)+\int_{0}^{1} J^{\prime}\left(-1+\theta\left(p_{c w}+1\right), \theta p_{c g}\right)\left(p_{c w}+1, p_{c g}\right) \mathrm{d} \theta .
$$

Par définition de la fonction $J$ (voir (2.7)) et si on note

$$
\boldsymbol{z}=\left(z_{1}, z_{2}\right) \in \mathbb{R}^{2},
$$

on a

$$
\begin{aligned}
J^{\prime}(\boldsymbol{z}) & =\left(\begin{array}{c}
\frac{\partial J}{\partial p_{c w}}(\boldsymbol{z}) \\
\frac{\partial J}{\partial p_{c g}}(\boldsymbol{z})
\end{array}\right)=\left(\begin{array}{ll}
\frac{\partial \tilde{s}_{w}}{\partial p_{c w}}(\boldsymbol{z}) & \frac{\partial \tilde{s}_{g}}{\partial p_{c w}}(\boldsymbol{z}) \\
\frac{\partial \tilde{s}_{w}}{\partial p_{c g}}(\boldsymbol{z}) & \frac{\partial \tilde{s}_{g}}{\partial p_{c g}}(\boldsymbol{z})
\end{array}\right)\left(\begin{array}{l}
z_{1} \\
z_{2}
\end{array}\right) \\
& =\nabla_{p} \tilde{s}(\boldsymbol{z}) \boldsymbol{z} .
\end{aligned}
$$


Ainsi l'équation (5.27) nous permet d'écrire

$$
\begin{aligned}
J\left(p_{c w}, p_{c g}\right)= & \int_{0}^{1} \nabla_{p} \tilde{s}\left(-1+\tau\left(p_{c w}+1\right), \tau p_{c g}\right) \times \\
& \times\left(-1+\tau\left(p_{c w}+1\right), \tau p_{c g}\right)\left(p_{c w}+1, p_{c g}\right) \mathrm{d} \tau .
\end{aligned}
$$

D'après la convexité de la fonction $\Theta$ introduite dans la remarque 1 de la section 2 et de l'égalité ci-dessus, il existe une constante $\theta$ telle que

$$
\begin{aligned}
& \theta\left(\left|p_{c w}+1\right|^{2}+\left|p_{c g}\right|^{2}\right) \leq \\
& \quad \leq J\left(p_{c w}, p_{c g}\right)+\mid \int_{0}^{1} \nabla_{p} \tilde{s}\left(-1+\tau\left(p_{c w}+1\right), \tau p_{c g}\right) \times \\
& \quad \times\left(-1+(\tau-1)\left(p_{c w}+1\right),(\tau-1) p_{c g}\right)\left(p_{c w}+1, p_{c g}\right) \mathrm{d} \tau \mid .
\end{aligned}
$$

D'après l'hypothèse (H9) et l'inégalité de Young, on a

$$
\left|p_{c w}+1\right|^{2}+\left|p_{c g}\right|^{2} \leq \frac{2}{\theta} J\left(p_{c w}, p_{c g}\right)+c_{12},
$$

où $c_{12}$ est une constante.

De cette estimation et de (5.22), on déduit alors que

$$
\left(p_{c w}, p_{c g}\right) \text { est borné dans }\left(L ^ { \infty } \left(\left[0, t\left[\times L^{2}(\Omega)\right)\right)^{2} .\right.\right.
$$

3. Estimation sur $\partial_{t}\left(\boldsymbol{\Phi} \tilde{\boldsymbol{s}}_{w}\right)$ et $\partial_{t}\left(\boldsymbol{\Phi} \tilde{\boldsymbol{s}}_{g}\right)$

On utilise (5.10) pour écrire l'inégalité suivante $\forall u \in L^{2}([0, t[, \mathcal{V})$ :

$$
\begin{aligned}
\left|\left\langle\partial_{t}\left(\boldsymbol{\Phi}(x) \tilde{\boldsymbol{s}}_{w}\left(\boldsymbol{p}_{c}\right)\right), u\right\rangle\right| \leq \\
\leq\left|\tilde{\nu}_{w}\right|_{L^{\infty}(\Omega)}\left|\boldsymbol{V}_{T}\right|_{L^{2}(\Omega)}|\nabla u|_{L^{2}(\Omega)}+ \\
\quad+\left|\mathbf{K}(x) \widetilde{\bar{M}}_{g}\right|_{L^{\infty}(\Omega)}\left|\nabla p_{c w}\right|_{L^{2}(\Omega)}|\nabla u|_{L^{2}(\Omega)}+ \\
\quad+\left|\mathbf{K}(x) \widetilde{\bar{M}}_{o}\right|_{L^{\infty}(\Omega)}\left|\nabla p_{c w}-\nabla p_{c g}\right|_{L^{2}(\Omega)}|\nabla u|_{L^{2}(\Omega)}+ \\
\quad+\left|\mathbf{K}(x) \tilde{\bar{\rho}}_{\omega} \boldsymbol{g}\right|_{L^{\infty}(\Omega)}(\operatorname{mes}(\Omega))|\nabla u|_{L^{2}(\Omega)}+ \\
\quad+\varepsilon\left|\nabla \tilde{s}_{w}\left(\boldsymbol{p}_{c}\right)\right|_{L^{2}(\Omega)}|\nabla u|_{L^{2}(\Omega)}+ \\
\quad+\left|\tilde{\nu}_{w}\right|_{L^{2}\left(\Gamma_{s}\right)}\left|q_{s}\right|_{L^{2}\left(\Gamma_{s}\right)}|u|_{L^{2}\left(\Gamma_{s}\right)}
\end{aligned}
$$


En tenant compte des estimations $\left(5.13^{\prime}\right),(5.20)$ et $(5.21)$, on déduit de (5.30) qu'il existe une constante $c_{13}(\varepsilon)$ dépendant des constantes $c_{1}-c_{7}$ telle que

$$
\left|\partial_{t}\left(\boldsymbol{\Phi}(x) \tilde{\boldsymbol{s}}_{\omega}\left(\boldsymbol{p}_{c}\right)\right)\right|_{L^{2}\left(0, t, \mathcal{V}^{\prime}\right)} \leq c_{13}(\varepsilon) .
$$

De même, il existe une constante $c_{14}(\varepsilon)$ telle que

$$
\left|\partial_{t}\left(\boldsymbol{\Phi}(x) \tilde{s}_{g}\left(\boldsymbol{p}_{c}\right)\right)\right|_{L^{2}\left(0, t, \mathcal{V}^{\prime}\right)} \leq c_{14}(\varepsilon) .
$$

Cela termine la démonstration de la proposition 1.

\section{Étape 2. Passage à la limite}

Le passage à la limite, lorsque $h$ tend vers zéro, se fait grâce aux arguments classiques de compacité associés au lemme d'Aubin et à l'hypothèse de prolongement (H9).

On va tout d'abord obtenir des estimations sur les saturations en utilisant l'estimation (5.29). On a

$$
\begin{aligned}
\tilde{\boldsymbol{s}}_{w}\left(p_{c w}^{h}, p_{c g}^{h}\right)= & \tilde{\boldsymbol{s}}_{w}(-1,0)+ \\
& +\int_{0}^{1} \frac{\partial \tilde{\boldsymbol{s}}_{w}}{\partial p_{c w}}\left(-1+\theta\left(p_{c w}^{h}+1\right), \theta p_{c g}^{h}\right)\left(p_{c w}^{h}+1\right) \mathrm{d} \theta+ \\
& +\int_{0}^{1} \frac{\partial \tilde{s}_{w}}{\partial p_{c w}}\left(-1+\theta\left(p_{c w}^{h}+1\right), \theta p_{c g}^{h}\right) p_{g}^{h} \mathrm{~d} \theta .
\end{aligned}
$$

Ainsi, en tenant compte de (H9), on a

$$
\left|\tilde{s}_{w}\left(\boldsymbol{p}_{c}^{h}\right)\right| \leq s_{w}^{2}\left|p_{c w}^{h}+1\right|+\left|s_{w}^{4}\right|\left|p_{c g}^{h}\right| .
$$

De l'estimation (5.29) et de celle ci-dessus, on déduit que

$$
\begin{gathered}
\tilde{s}_{w}\left(\boldsymbol{p}_{c}^{h}\right) \text { est borné dans } L^{\infty}\left(\left[0, t\left[, L^{2}(\Omega)\right)\right.\right. \text { par une constante } \\
\text { indépendante de } h
\end{gathered}
$$

De plus, d'après l'hypothèse $(\mathrm{H} 9)$ et $(5.15)$, on a

$$
\begin{aligned}
\left|\nabla \tilde{\boldsymbol{s}}_{w}\left(\boldsymbol{p}_{c}^{h}\right)\right|_{L^{2}(\Omega)} \leq & \left|\frac{\partial \tilde{\boldsymbol{s}}_{w}}{\partial p_{c w}}\left(\boldsymbol{p}_{c}^{h}\right)\right|_{L^{\infty}(\Omega)}\left|\nabla p_{c w}^{h}\right|_{L^{2}(\Omega)}+ \\
& +\left|\frac{\partial \tilde{\boldsymbol{s}}_{w}}{\partial p_{c g}}\left(\boldsymbol{p}_{c}^{h}\right)\right|_{L^{\infty}(\Omega)}\left|\boldsymbol{\nabla} p_{c g}^{h}\right|_{L^{2}(\Omega)} \\
& -\mathbf{3 5 8}-
\end{aligned}
$$


En tenant compte de (5.33), (5.20) et (5.21), on déduit que

$$
\tilde{s}_{w}\left(\boldsymbol{p}_{c}^{h}\right) \text { est bornée dans } L^{\infty}\left(\left[0, t\left[, L^{2}(\Omega)\right) \cap L^{2}\left(\left[0, t\left[, H^{1}(\Omega)\right) .\right.\right.\right.\right.
$$

Maintenant, on établit une convergence forte des saturations dans $L^{2}\left(\left[0, T\left[, L^{2}(\Omega)\right)\right.\right.$.

On a $\boldsymbol{p}_{c}^{h}=0$ sur $\Gamma_{e}$, ainsi $\tilde{s}\left(\boldsymbol{p}_{c}^{h}\right) \Gamma_{\Gamma_{e}}=1=s_{w}^{D}(x)$.

Par suite, l'estimation (5.34) est équivalente à

$$
\begin{gathered}
\tilde{s}_{w}\left(\boldsymbol{p}_{c}^{h}\right) \text { est bornée dans } \\
L^{\infty}\left(\left[\mathbf{0}, t\left[, L^{2}(\Omega)\right) \cap L^{2}\left(\left[0, t\left[,\left\{u \in H^{1}(\Omega) \mid u=s_{w}^{D}(x) \operatorname{sur} \Gamma_{e}\right\}\right) .\right.\right.\right.\right.
\end{gathered}
$$

On note encore $s_{w}^{D}(x)$ le relèvement de $s_{w}^{D}(x) \Gamma_{\Gamma_{e}}$ sur $H^{1}(\Omega)$.

On a donc

$$
\bar{s}_{w}^{h}=\tilde{s}_{w}\left(p_{c}^{h}\right)-s_{w}^{D}(x) \text { est borné dans } L^{2}([0, t[, \mathcal{V}),
$$

et d'après $(5.31)$ on a

$$
\partial_{t}\left(\boldsymbol{\Phi} \bar{s}_{w}^{h}\right) \text { est borné dans } L^{2}\left(\left[0, t\left[, \mathcal{V}^{\prime}\right) .\right.\right.
$$

LeMMe 1. - Soit $\mathbf{\Phi}$ vérifiant (H7);

alors l'injection de $\mathcal{W}$ dans $L^{2}\left(\left[0, t\left[, L^{2}(\Omega)\right)\right.\right.$ est compacte, où

$$
\mathcal{W}=\left\{u \mid u \in L^{2}\left(\left[0, t[, \mathcal{V}) ; \partial_{t}(\Phi(x) u) \in L^{2}\left(\left[0, t\left[, \mathcal{V}^{\prime}\right)\right\}\right.\right.\right.\right.
$$

Preuve. - Comme $\Phi$ est de classe $C^{1}$, alors l'application $u \rightarrow \Phi u=v$ est continue de $L^{2}\left(\left[0, t[, \mathcal{V})\right.\right.$ dans $L^{2}([0, t[, \mathcal{V})$.

Ensuite d'après le lemme d'Aubin (voir par exemple [18]), on sait que l'injection de

$$
\left\{v / v \in L^{2}\left(\left[0, t[, \mathcal{V}) ; \partial_{t} v \in L^{2}\left(\left[0, t\left[, \mathcal{V}^{\prime}\right)\right\}\right.\right.\right.\right.
$$

dans $L^{2}\left(\left[0, t\left[, L^{2}(\Omega)\right)\right.\right.$ est compacte.

On déduit le résultat annoncé du fait que l'application $v \rightarrow(v / \Phi)=u$ est continue de $L^{2}\left(\left[0, t\left[, L^{2}(\Omega)\right)\right.\right.$ dans $L^{2}\left(\left[0, t\left[, L^{2}(\Omega)\right)\right.\right.$. 
De (5.35), (5.36) et du lemme 1 , on peut supposer quitte à extraire une sous-suite que

$$
\bar{s}_{w}^{h} \text { converge fortement dans } L^{2}\left(\left[0, t\left[, L^{2}(\Omega)\right) .\right.\right.
$$

Ceci entraîne

$$
\tilde{s}_{w}^{h}=\tilde{s}_{w}\left(\boldsymbol{p}_{c}^{h}\right) \rightarrow \tilde{s}_{w} \text { dans } L^{2}\left(\left[\mathbf{0}, t\left[, L^{2}(\Omega)\right) \text { et p.p. dans } \Omega .\right.\right.
$$

De même,

$$
\tilde{s}_{g}^{h}=\tilde{s}_{g}\left(\boldsymbol{p}_{c}^{h}\right) \rightarrow \tilde{s}_{g} \text { dans } L^{2}\left(\left[0, t\left[, L^{2}(\Omega)\right) \text { et p.p. dans } \Omega .\right.\right.
$$

Des estimations $(5.20)$ et $(5.21)$, on a

$$
\begin{aligned}
p_{c w}^{h} & \rightarrow F_{w} \text { dans } L^{2}([0, t[, \mathcal{V}) \text { faiblement } \\
p_{c g}^{h} & \rightarrow F_{g} \text { dans } L^{2}([0, t[, \mathcal{V}) \text { faiblement. }
\end{aligned}
$$

Nous allons maitenant définir la relation entre les limites $\left(s_{w}, s_{g}\right)$ et $\left(F_{w}, F_{g}\right)$.

En effet, les hypothèses (H1) et (H9) assurent l'inversibilité des saturations en fonction des pressions capillaires, par conséquent

$$
p_{c w}^{h}=G_{w}\left(\tilde{s}_{w}^{h}, \tilde{s}_{g}^{h}\right) \quad \text { et } \quad p_{c g}^{h}=G_{g}\left(\tilde{s}_{w}^{h}, \tilde{s}_{g}^{h}\right)
$$

De plus, toutes les dérivées partielles des pressions capillaires par rapport aux saturations sont uniformément bornées. Ceci entraîne que $G_{w}$ et $G_{g}$ sont uniformément lipschitziennes en $\tilde{s}_{w}$ et $\tilde{s}_{g}$, et d'après (5.37) et (5.38) on a :

$$
G_{w}\left(\widetilde{s}_{w}^{h}, \tilde{s}_{g}^{h}\right) \rightarrow G_{w}\left(\tilde{s}_{w}, \tilde{s}_{g}\right) \text { dans } L^{2}\left(\left[0, t\left[, L^{2}(\Omega)\right)\right.\right. \text { fortement }
$$

et

$$
G_{g}\left(\tilde{s}_{w}^{h}, \tilde{s}_{g}^{h}\right) \rightarrow G_{g}\left(\tilde{s}_{w}, \tilde{s}_{g}\right) \text { dans } L^{2}\left(\left[0, t\left[, L^{2}(\Omega)\right)\right.\right. \text { fortement. }
$$

Des estimations (5.39)-(5.40) et de celles ci-dessus, on déduit que

$$
G_{w}\left(\tilde{s}_{w}, \tilde{s}_{g}\right)=F_{w}, \quad G_{g}\left(\tilde{s}_{w}, \tilde{s}_{g}\right)=F_{g}
$$


En utilisant $(5.37)$ et $(5.38)$ et le fait que $\tilde{\nu}_{\eta}, \widetilde{M}$ sont des fonctions continues en $\tilde{s}_{w}$ et $\tilde{s}_{g}$, on a d'après le théorème de convergence dominée de Lebesgue

$$
\begin{gathered}
\tilde{\nu}_{\eta}\left(\widetilde{s}_{w}^{h}, \tilde{s}_{g}^{h}\right) \rightarrow \tilde{\nu}_{\eta}\left(\tilde{s}_{w}, \tilde{s}_{g}\right) \text { fortement dans } L^{p}\left(\left[0, t\left[, L^{p}(\Omega)\right)\right.\right. \\
\text { pour tout } p \text { fini }
\end{gathered}
$$

et

$$
\begin{aligned}
\widetilde{M}\left(\widetilde{s}_{w}^{h}, \widetilde{s}_{g}^{h}\right) \rightarrow & \widetilde{M}\left(\tilde{s}_{w}, \tilde{s}_{g}\right) \text { fortement dans } L^{p}\left(\left[0, t\left[, L^{p}(\Omega)\right)\right.\right. \\
& \text { pour tout } p \text { fini. }
\end{aligned}
$$

Compte tenu de (5.37)-(5.43) et du fait que les translations sont uniformément continues dans $L^{p}(0, T)$ pour tout $p$ fini, le passage à la limite en $h$ sur le problème linéarisé défini par l'algorithme $(A)$ ne révèle pas de difficulté. On déduit l'existence d'une solution pour le problème $\left(P_{\varepsilon}\right)$.

Pour terminer la démonstration du théorème 1 , nous allons prouver que les saturations appartiennent au convexe $\pi$.

Étape 3. Estimation $L^{\infty}\left(\mathbf{R}^{+} \times \Omega\right)$

Nous allons démontrer le résultat suivant.

Proposition 2. $-S i\left(s_{w}^{0}(x), s_{g}^{0}(x)\right)$ appartient $a$ T alors : $\left(s_{w}(t, x), s_{g}(t, x)\right) \in \pi$ pour presque tout $t \in[0, T[$.

Preuve. - Pour cela on va tout d'abord définir le prolongement des fonctions $\nu_{w}, \nu_{o}, \nu_{g}$ et $M$ en dehors de leur domaine de définition, à savoir le convexe $\pi$ en des fonctions $\tilde{\nu}_{w}, \tilde{\nu}_{o}, \widetilde{\nu}_{g}$ et $\widetilde{M}$ continues et bornées sur $\mathbb{R} \times \mathbb{R}$.

Cette étape est essentielle parce qu'on ne sait pas a priori que les saturations sont admissibles.

Dans [12], un prolongement continu de $\nu_{\eta}, \eta=w, o, g$ et $M$ a été explicité; il consiste à définir $\tilde{\nu}_{\eta}, \eta=w, o, g$ et $\widetilde{M}$, en dehors de $\pi$, de la manière suivante :

$$
\tilde{\nu}_{w}(x, y)= \begin{cases}0 & \text { si } x \leq 0 \\ \nu_{w}(x, 1-x) & \text { si } 0 \leq x \leq 1 \text { et } x+y \geq 1 \\ \nu_{w}(x, 0) & \text { si } 0 \leq x \leq 1 \text { et } y \leq 0 \\ 1 & \text { si } x \geq 1\end{cases}
$$




$$
\begin{aligned}
& \text { Pierre Fabrie et Mazen Saad } \\
& \tilde{\nu}_{g}(x, y)= \begin{cases}\nu_{g}(0, y) & \text { si } 0 \leq 0 \text { et } 0 \leq y \leq 1 \\
0 & \text { si } y \leq 0 \text { ou } x \geq 1 \\
1 & \text { si } x \leq 0 \text { et } y \geq 1 \\
\nu_{g}(x, 1-x) & \text { si } 0 \leq x \leq 1 \text { et } x+y \geq 1\end{cases} \\
& \tilde{\nu}_{o}=1-\tilde{\nu}_{w}-\tilde{\nu}_{g} .
\end{aligned}
$$

$\widetilde{M}$ est définie en dehors de $\pi$ de la manière suivante : soient $\boldsymbol{x}$ un point donné intérieur à $\boldsymbol{\pi}$ et $\boldsymbol{y}=\left(y_{1}, y_{2}\right)$ un point quelconque à l'extérieur de $\boldsymbol{\pi}$. Soit alors $z=\left(z_{1}, z_{2}\right)$ le point d'intersection de $\partial \pi$ et du segment $x y$, on pose

$$
\widetilde{M}\left(y_{1}, y_{2}\right)=M\left(z_{1}, z_{2}\right)
$$

Les fonctions $s_{\eta}\left(\boldsymbol{p}_{c}\right)$ sont prolongées en des fonctions $\tilde{s}_{\eta}\left(\boldsymbol{p}_{c}\right)$ comme indiqué à l'étape 1 .

On récrit le problème régularisé $\left(P_{\varepsilon}\right)$ avec les fonctions prolongées :

$$
\begin{aligned}
& \left\langle\Phi(x) \partial_{t} \tilde{s}_{w}\left(p_{c}\right), u\right\rangle-\int_{\Omega} \tilde{\nu}_{w} V_{T} \cdot \nabla u \mathrm{~d} \omega+ \\
& +\int_{\Omega} \mathbf{K}(x) \widetilde{M} \tilde{\nu}_{w} \tilde{\nu}_{o} \nabla p_{c w} \cdot \nabla u \mathrm{~d} \omega+\varepsilon \int_{\Omega} \nabla \tilde{s}_{w}\left(p_{c}\right) \cdot \nabla u \mathrm{~d} \omega+ \\
& +\int_{\Omega} \mathbf{K}(x) \widetilde{M} \tilde{\nu}_{w} \tilde{\nu}_{g}\left(\nabla p_{c w}-\nabla p_{c g}\right) \cdot \nabla u \mathrm{~d} \omega+ \\
& -\int_{\Omega} \mathbf{K}(x) \widetilde{M} \tilde{\nu}_{w}\left(\tilde{\nu}_{o}\left(\rho_{w}-\rho_{o}\right)+\tilde{\nu}_{g}\left(\rho_{w}-\rho_{g}\right)\right) \boldsymbol{g} \cdot \nabla u \mathrm{~d} \boldsymbol{\omega}= \\
& =-\int_{\Gamma_{s}} \tilde{\nu}_{w} q_{s} u \mathrm{~d} \gamma_{s}, \quad \forall u \in V, \quad \text { p.p. dans }[0, t[ \\
& \left\langle\Phi(x) \partial_{t} \tilde{s}_{g}\left(\boldsymbol{p}_{c}\right), v\right\rangle-\int_{\Omega} \tilde{\nu}_{g} V_{T} \cdot \nabla v \mathrm{~d} \omega+ \\
& +\int_{\Omega} \mathbf{K}(x) \widetilde{M} \tilde{\nu}_{g} \tilde{\nu}_{o} \nabla p_{c g} \cdot \nabla v \mathrm{~d} \boldsymbol{\omega}+\varepsilon \int_{\Omega} \nabla \tilde{s}_{g}\left(\boldsymbol{p}_{c}\right) \cdot \nabla v \mathrm{~d} \boldsymbol{\omega}+ \\
& +\int_{\Omega} \mathbf{K}(x) \widetilde{M} \tilde{\nu}_{w} \tilde{\nu}_{g}\left(\nabla p_{c g}-\nabla p_{c w}\right) \cdot \nabla v \mathrm{~d} \omega+ \\
& -\int_{\Omega} \mathbf{K}(x) \widetilde{M} \tilde{\nu}_{g}\left(\tilde{\nu}_{o}\left(\rho_{g}-\rho_{o}\right)+\tilde{\nu}_{w}\left(\rho_{g}-\rho_{w}\right)\right) \boldsymbol{g} \cdot \nabla v \mathrm{~d} \omega= \\
& =-\int_{\Gamma_{s}} \tilde{\nu}_{g} q_{s} v \mathrm{~d} \gamma_{s}, \quad \forall v \in V, \quad \text { p.p. dans }[0, t[
\end{aligned}
$$


$-\int_{\Omega} V_{T} \cdot \nabla \psi \mathrm{d} \omega=\int_{\Gamma_{s}} q_{e} \psi \mathrm{d} \gamma_{s}-\int_{\Gamma_{s}} q_{s} \psi \mathrm{d} \gamma_{s}, \quad \forall \psi \in H^{1}(\Omega) / \mathbb{R}$.

$V_{T}$ est définie par (3.3).

i) Montrons que $\tilde{s}_{w} \leq 1$

On a par construction du prolongement :

$$
\tilde{\nu}_{w}=1 \text { si } \tilde{s}_{w} \geq 1 ; \quad \tilde{\nu}_{g}=\tilde{\nu}_{o}=0 \text { si } \tilde{s}_{w} \geq 1
$$

En prenant $u=\left(\tilde{s}_{w}-1\right)^{+}$dans $(5.44)$, on obtient

$$
\begin{aligned}
& \left\langle\Phi(x) \partial_{t} \tilde{s}_{w},\left(\tilde{s}_{w}-1\right)^{+}\right\rangle-\int_{\Omega} V_{T} \nabla\left(\tilde{s}_{w}-1\right)^{+} \mathrm{d} \omega+ \\
& \quad+\varepsilon \int_{\Omega} \nabla \tilde{s}_{w} \nabla\left(\tilde{s}_{w}-1\right)^{+} \mathrm{d} \omega=-\int_{\Gamma_{s}} q_{s}\left(\tilde{s}_{w}-1\right)^{+} \mathrm{d} \gamma_{e} .
\end{aligned}
$$

Compte tenu de (5.46) pour $\psi=\left(\tilde{s}_{w}-1\right)^{+}$, on a

$$
-\int_{\Omega} V_{T} \nabla\left(\tilde{s}_{w}-1\right)^{+} \mathrm{d} \omega=\int_{\Gamma_{e}} q_{e}\left(\tilde{s}_{w}-1\right)^{+} \mathrm{d} \gamma_{e}-\int_{\Gamma_{s}} q_{s}\left(\tilde{s}_{w}-1\right)^{+} \mathrm{d} \gamma_{s} ;
$$

par suite $(5.47)$ se réduit à

$$
\begin{gathered}
\left\langle\Phi(x) \partial_{t}\left(\tilde{s}_{w}-1\right)^{+},\left(\tilde{s}_{w}-1\right)^{+}\right\rangle+\varepsilon \int_{\Omega}\left|\nabla\left(\tilde{s}_{w}-1\right)^{+}\right|^{2} \mathrm{~d} \omega= \\
=-\int_{\Gamma_{e}} q_{e}\left(\tilde{s}_{w}-1\right)^{+} \mathrm{d} \gamma_{e} .
\end{gathered}
$$

Ceci entraîne

$$
\frac{\mathrm{d}}{\mathrm{d} t}\left|\left(\tilde{s}_{w}-1\right)^{+}\right|_{L^{2}(\Omega)}^{2} \leq 0 .
$$

En intégrant entre 0 et $t$ la dernière inégalité, on obtient

$$
\int_{\Omega}\left(\left|\left(\tilde{s}_{w}-1\right)^{+}(t)\right|^{2}-\left|\left(\tilde{s}_{w}^{0}-1\right)^{+}\right|^{2}\right) \mathrm{d} \omega \leq 0 .
$$

Or $0 \leq s_{w}^{0} \leq 1$, donc $\left(s_{w}^{0}-1\right)^{+}=0$ presque partout dans $\Omega$. Par conséquent

$$
\tilde{s}_{w} \leq 1 \text { p.p. dans } \Omega \text {. }
$$


ii) Montrons que $\tilde{s}_{\eta} \geq 0, \eta=w, g$

On a par construction $\tilde{\nu}_{\eta}=0$ si $\tilde{s}_{\eta} \leq 0$. En prenant $u=-\tilde{s}_{w}^{-}$dans (5.44) et $v=-\tilde{s}_{g}^{-}$dans (5.45), on déduit d'une manière indentique à l'étape i) :

$$
\tilde{s_{\eta}}=0 \quad \text { p.p. dans } \Omega \text { pour } \eta=w, g .
$$

iii) Montrons que $\tilde{s}_{g} \leq 1$ et $0 \leq 1-\tilde{s}_{w}-\tilde{s}_{g} \leq 1$

On a vu que $0 \leq s_{w} \leq 1$ et $\tilde{s}_{g} \geq 0$, il reste à montrer que $\tilde{s}_{w}+\tilde{s}_{g} \leq 1$.

On a par construction du prolongement dans la région où $0 \leq \tilde{s}_{w} \leq 1$, $\tilde{s}_{g} \geq 0$ et $\tilde{s}_{w}+\tilde{s}_{g} \geq 1:$

$$
\tilde{\nu}_{o}\left(s_{w}, \tilde{s}_{g}\right)=0
$$

Pour mieux tenir compte de cette remarque, l'idée est de travailler avec l'équation de conservation de l'huile écrite sous forme variationnelle. Pour cela, on prend $u=v=\psi$ dans (5.44)-(5.46) et en sommant ces trois équtions, on obtient

$$
\begin{aligned}
\left\langle\Phi(x) \partial_{t}\left(\tilde{s}_{w}+\tilde{s}_{g}-1\right), \psi\right\rangle+\int_{\Omega} \tilde{\nu}_{o} V_{T} \nabla \psi \mathrm{d} \omega+ \\
\quad+\int_{\Omega} \mathbf{K}(x) \widetilde{M} \tilde{\nu}_{o} \tilde{\nu}_{w} \nabla p_{c w} \cdot \nabla \psi \mathrm{d} \omega+\int_{\Omega} \mathbf{K}(x) \widetilde{M} \tilde{\nu}_{o} \tilde{\nu}_{g} \nabla p_{c g} \cdot \nabla \psi \mathrm{d} \omega+ \\
\quad+\varepsilon \int_{\Omega} \nabla\left(\tilde{s}_{w}+\tilde{s}_{g}-1\right) \cdot \nabla \psi \mathrm{d} \omega+ \\
\quad-\int_{\Omega} \mathbf{K}(x) \widetilde{M} \tilde{\nu}_{o}\left(\tilde{\nu}_{o}\left(\rho_{w}-\rho_{o}\right)+\tilde{\nu}_{g}\left(\rho_{g}-\rho_{o}\right) \boldsymbol{g} \cdot \nabla \psi \mathrm{d} \omega=\right. \\
=-\int_{\Gamma_{e}} q_{e} \psi \mathrm{d} \gamma_{e}+\int_{\Gamma_{s}} \tilde{\nu}_{o} q_{s} \psi \mathrm{d} \gamma_{s} .
\end{aligned}
$$

En prenant $\psi=\left(\tilde{s}_{w}+\tilde{s}_{g}-1\right)^{+}$dans l'égalité ci-dessous, on obtient d'une manière identique à $i$ ):

$$
\left(\tilde{s}_{w}+\tilde{s}_{g}-1\right)^{+}=0 \quad \text { p.p. dans } \Omega .
$$

Ceci termine la démonstration du théorème $1 . \square$ 


\section{Existence d'une solution faible pour le problème dégénéré}

Nous proposons ici de démontrer un résultat d'existence d'une solution faible pour le système dégénéré.

Dans [6]-[7] pour un problème diphasique, G. Chavent définit des fonctions höldériennes afin d'absorber la dégénérescence du système. Le passage à la limite s'obtient alors en utilisant des arguments classiques de compacité associés à la caractérisation de Gagliardo des espaces $W^{s, p}(\Omega)$ pour $0<s<1,1<p<\infty$.

Dans [4] pour un problème diphasique, T. Arbogast, en faisant intervenir la notion de pression globale et en définissant une fonction dont le gradient absorbe les termes elliptiques dégénérés, obtient un système non dégénéré. En supposant essentiellement que la saturation est une fonction croissante de cette dernière fonction, il montre l'existence d'une solution faible.

Nous allons donner une condition suffisante pour que les termes elliptiques dégénérés soient absorbés par un changement de fonction. Cette hypothèse conduit à un système aux dérivées partielles liant les fractions de flux aux pressions capillaires et par conséquent à un modèle de perméabilités relatives.

Plus précisément, on suppose dans ce paragraphe l'existence d'un facteur intégrant $\mathbf{A}$ définit de $\mathbb{R}^{2}$ dans $\mathbb{R}^{2}$ vérifiant les hypothèses suivantes.

$(\mathrm{H} 10)_{1} \mathbf{D}\left(\boldsymbol{p}_{c}\right)=\mathbf{B}\left(\boldsymbol{p}_{c}\right) \boldsymbol{\nabla} \mathbf{A}$, où $\boldsymbol{\nabla} \mathbf{A}$ désigne la jacobienne de $\mathbf{A}$ dans les variables $p_{c}$, et $\mathbf{D}$ est définie par

$$
\mathbf{D}=\mathbf{M}\left[\begin{array}{cc}
\nu_{w}\left(1-\nu_{w}\right) & -\nu_{w} \nu_{g} \\
-\nu_{w} \nu_{g} & \nu_{g}\left(1-\nu_{g}\right)
\end{array}\right] \text {. }
$$

$(\mathrm{H} 10)_{2} \mathbf{B}\left(\boldsymbol{p}_{c}\right)=\left(b_{i j}\left(\boldsymbol{p}_{c}\right)\right)$ est un opérateur continu sur son domaine et vérifie

$$
\exists b \in \mathbb{R}_{+}^{*} \text { tel que } \forall \boldsymbol{\xi} \in \mathbb{R}^{2}, \quad \mathbf{B}\left(\boldsymbol{p}_{c}\right) \cdot \boldsymbol{\xi} \cdot \boldsymbol{\xi} \geq b|\boldsymbol{\xi}|^{2} .
$$

(H10) ${ }_{3} \mathbf{A}^{-1}$ est höldérienne d'ordre $\theta, 0<\theta<1$, où $\mathbf{A}$ est la fonction de $\mathbb{R}^{2}$ dans $\mathbb{R}^{2}$ définie par

$$
\mathbf{A}\left(\boldsymbol{p}_{c}\right)=\left(\begin{array}{l}
f\left(\boldsymbol{p}_{c}\right) \\
g\left(\boldsymbol{p}_{c}\right)
\end{array}\right)
$$


Le sens donné à l'hypothèse $(\mathrm{H} 10)_{3}$ est le suivant : si on note

$$
\mathbf{A}^{-1}\left(\begin{array}{c}
f \\
g
\end{array}\right)=\left(\begin{array}{c}
u_{w}(f, g) \\
u_{g}(f, g)
\end{array}\right)=\left(\begin{array}{c}
p_{c w} \\
p_{c g}
\end{array}\right) .
$$

$\mathbf{A}^{-1}$ est höldérienne d'ordre $\theta$ si et seulement si

$$
\begin{gathered}
\forall(a, c) \in \mathbb{R}, \exists\left(c_{1}^{\eta}, c_{2}^{\eta}\right) \in \mathbb{R}_{+} \times \mathbb{R}_{+}, \forall\left(x_{1}, y_{1}, x_{2}, y_{2}\right) \in[a, c]^{4} \\
\left|u_{\eta}\left(x_{1}, y_{1}\right)-u_{\eta}\left(x_{2}, y_{2}\right)\right| \leq c_{1}^{\eta}\left|x_{1}-x_{2}\right|^{\theta}+c_{2}^{\eta}\left|y_{1}-y_{2}\right|^{\theta} \quad \text { pour } \eta=w, g .
\end{gathered}
$$

$(\mathrm{H} 10)_{4}$ Il existe une fonction $q$ définie de $\mathbb{R}^{2}$ dans $\mathbb{R}_{+}$vérifiant

$$
\partial_{t} \boldsymbol{s}\left(\boldsymbol{p}_{c}\right) \cdot \mathbf{A}\left(\boldsymbol{p}_{c}\right)=\partial_{t} q\left(\boldsymbol{p}_{c}\right)
$$

Remarque 2. - $\grave{\mathbf{A}}$ titre d'exemple un choix de $\mathbf{B}$ et $\mathbf{A}$ est le suivant

$$
\mathbf{B}\left(\boldsymbol{p}_{c}\right)=\mathbf{M}\left(\boldsymbol{p}_{c}\right) \mathbf{I} .
$$

Ce choix de $\mathbf{B}\left(\boldsymbol{p}_{c}\right)$ vérifie $(\mathrm{H} 10)_{2}$.

L'existence, dans ce cas, d'une fonction $\mathbf{A}\left(\boldsymbol{p}_{c}\right)$ est assurée par le théorème de Poincaré et l'hypothèse $(\mathrm{H} 10)_{1}$ à condition que les fractions des flux vérifient les deux relations ci-dessous :

$$
\begin{gathered}
\frac{\partial\left(\nu_{w}\left(1-\nu_{w}\right)\right)}{\partial p_{c g}}+\frac{\partial\left(\nu_{w} \nu_{g}\right)}{\partial p_{c w}}=0 ; \\
\frac{\partial\left(\nu_{g}\left(1-\nu_{g}\right)\right)}{\partial p_{c w}}+\frac{\partial\left(\nu_{w} \nu_{g}\right)}{\partial p_{c g}}=0 .
\end{gathered}
$$

L'hypothèse $(\mathrm{H} 10)_{4}$ s'écrit alors

$$
\nu_{g}\left(1-\nu_{g}\right) \frac{\partial s_{g}}{\partial p_{c w}}-\nu_{w} \nu_{g} \frac{\partial s_{w}}{\partial p_{c w}}=\nu_{w}\left(1-\nu_{w}\right) \frac{\partial s_{w}}{\partial p_{c g}}-\nu_{w} \nu_{g} \frac{\partial s_{g}}{\partial p_{c g}} .
$$

Les hypothèses $(\mathrm{H} 10)_{1}$ et $(\mathrm{H} 3)$ conduisent à un modèle de mobilités, et les hypothèses $(\mathrm{H} 1)$ et $(\mathrm{H} 10)_{4}$ conduisent, quant à elles, à un modèle de saturations.

THÉORÈme 2. - Sous les hypothèses (H1)-(H8) et (H10), le système dégénéré $(P)$ admet au moins une solution faible. 
Existence de solutions faibles pour un modèle d'écoulement triphasique

Preuve. - Le système dégénéré $(\mathrm{P})$ est approché, sous l'hypothèse (H10), par une suite de problèmes non dégénérés $\left(P_{\varepsilon}\right)$ qui d'après le théorème 1 possède une solution $\left(p_{c w}^{\varepsilon}, p_{c g}^{\varepsilon}, V_{T}^{\varepsilon}, p^{\varepsilon}\right)$ vérifiant

$$
\begin{aligned}
& \left(s_{w}\left(\boldsymbol{p}_{c}^{\varepsilon}\right), s_{g}\left(\boldsymbol{p}_{c}^{\varepsilon}\right)\right) \in \pi \quad \text { p.p. dans } \Omega, \\
& \left(p_{c w}^{\varepsilon}, p_{c w}^{\varepsilon}\right) \in\left(L ^ { \infty } \left(\left[0, t\left[, L^{2}(\Omega)\right) \cap L^{2}\left([0, t[, \mathcal{V}))^{2},\right.\right.\right.\right. \\
& p^{\varepsilon} \in L^{\infty}\left(\left[0, t\left[, H^{1}(\Omega) / \mathbb{R}\right) ; \quad V_{T}^{\varepsilon} \in L^{\infty}([0, t[, \mathbb{H}) \text {. }\right.\right. \\
& \forall(u, v) \in L^{2}(0, T, \mathcal{V})^{2}: \\
& \left\langle\partial_{t}\left(\boldsymbol{\Phi}(x) s_{w}\left(\boldsymbol{p}_{c}^{\varepsilon}\right)\right), u\right\rangle-\int_{\Omega} \nu_{w}\left(\boldsymbol{s}\left(\boldsymbol{p}_{c}^{\varepsilon}\right)\right) \boldsymbol{V}_{T}^{\varepsilon} \cdot \nabla u \mathrm{~d} \omega+ \\
& +\varepsilon \int_{\Omega} \nabla s_{w}\left(p_{c}^{\varepsilon}\right) \cdot \nabla u \mathrm{~d} \omega+ \\
& +\int_{\Omega} \mathbf{K}(x)\left(b_{11}\left(\boldsymbol{p}_{c}^{\varepsilon}\right) \nabla f\left(\boldsymbol{p}_{c}^{\varepsilon}\right)+b_{12}\left(\boldsymbol{p}_{c}^{\varepsilon}\right) \nabla g\left(\boldsymbol{p}_{c}^{\varepsilon}\right)\right) \cdot \nabla u \mathrm{~d} \omega+ \\
& -\int_{\Omega} \mathbf{K}(x) \bar{\rho}_{w}\left(\boldsymbol{s}\left(\boldsymbol{p}_{c}^{\varepsilon}\right)\right) \boldsymbol{g} \cdot \nabla u \mathrm{~d} \boldsymbol{\omega}= \\
& =-\int_{\Gamma_{s}} \nu_{w}\left(s\left(p_{c}^{\varepsilon}\right)\right) q_{s} u \mathrm{~d} \gamma_{s} \\
& \left\langle\partial_{t}\left(\Phi(x) s_{g}\left(p_{c}^{\varepsilon}\right)\right), v\right\rangle-\int_{\Omega} \nu_{g}\left(s\left(p_{c}^{\varepsilon}\right)\right) V_{T}^{\varepsilon} \nabla v \mathrm{~d} \omega+ \\
& +\varepsilon \int_{\Omega} \nabla s_{g}\left(p_{c}^{\varepsilon}\right) \cdot \nabla v \mathrm{~d} \omega+ \\
& +\int_{\Omega} \mathbf{K}(x)\left(b_{21}\left(\boldsymbol{p}_{c}^{\varepsilon}\right) \boldsymbol{\nabla} f\left(\boldsymbol{p}_{c}^{\varepsilon}\right)+b_{22}\left(\boldsymbol{p}_{c}^{\varepsilon}\right) \nabla g\left(\boldsymbol{p}_{c}^{\varepsilon}\right)\right) \cdot \boldsymbol{\nabla} v \mathrm{~d} \boldsymbol{\omega}+ \\
& -\int_{\Omega} \mathbf{K}(x) \bar{\rho}_{g}\left(\boldsymbol{s}\left(p_{c}^{\varepsilon}\right)\right) \boldsymbol{g} \cdot \boldsymbol{\nabla} v \mathrm{~d} \boldsymbol{\omega}= \\
& =-\int_{\Gamma_{s}} \nu_{g}\left(s\left(p_{c}^{\varepsilon}\right)\right) q_{s} v \mathrm{~d} \gamma_{s} \text {. }
\end{aligned}
$$

$$
\begin{aligned}
\forall \psi \in H^{1}(\Omega) / \mathbb{R}: \\
\qquad \begin{aligned}
\int_{\Omega} \mathbf{K}(x) M\left(\boldsymbol{s}\left(\boldsymbol{p}_{c}^{\varepsilon}\right)\right) \nabla p^{\varepsilon} \cdot \nabla \psi \mathrm{d} \omega= & \int_{\Omega} \mathbf{K}(\boldsymbol{x}) \bar{\rho}\left(\boldsymbol{s}\left(\boldsymbol{p}_{c}^{\varepsilon}\right)\right) \nabla \psi \mathrm{d} \omega+ \\
& +\int_{\Gamma_{e}} q_{e} \psi \mathrm{d} \gamma_{e}-\int_{\Gamma_{s}} q_{s} \psi \mathrm{d} \gamma_{s}
\end{aligned}
\end{aligned}
$$

$-367-$ 
telles que

$$
\begin{aligned}
& \left\|p^{\varepsilon}\right\|_{H^{1}(\Omega) / \mathbb{R}} \leq c_{15} ; \quad\left|V_{T ! H}^{\varepsilon}\right|_{\mathbb{H}} \leq c_{16}, \\
& \varepsilon^{2} \int_{0}^{t} \int_{\Omega}\left|\nabla s_{w}\left(p_{c}^{\varepsilon}\right)\right|^{2} \mathrm{~d} \omega \mathrm{d} \tau \leq c_{17}, \\
& \varepsilon^{2} \int_{0}^{t} \int_{\Omega}\left|\nabla s_{g}\left(p_{c}^{\varepsilon}\right)\right|^{2} \mathrm{~d} \omega \mathrm{d} \tau \leq c_{18} .
\end{aligned}
$$

Afin de passer à la limite sur $\varepsilon$ dans le problème $\left(\mathrm{P}_{\varepsilon}\right)$, on établit des estimations semblables à celles obtenues dans l'étape 1 de la section 5 mais indépendantes de $\varepsilon$.

On va tout d'abord estimer $f$ et $g$. En prenant $u=f\left(\boldsymbol{p}_{c}^{\varepsilon}\right)$ dans (6.4) et $v=g\left(p_{c}^{\varepsilon}\right)$ dans (6.5) puis en sommant ces deux équations en tenant compte de $(6.7)$ et de l'hypothèse $(\mathrm{H} 10)_{2}$, on obtient :

$$
\begin{aligned}
& \left\langle\partial_{t}\left(\boldsymbol{\Phi}(\boldsymbol{x}) \boldsymbol{s}_{w}\left(\boldsymbol{p}_{c}^{\varepsilon}\right)\right), f\left(\boldsymbol{p}_{c}^{\varepsilon}\right)\right\rangle+\left\langle\partial_{t}\left(\boldsymbol{\Phi}(x) \boldsymbol{s}_{g}\left(\boldsymbol{p}_{c}^{\varepsilon}\right)\right), g\left(\boldsymbol{p}_{c}^{\varepsilon}\right)\right\rangle+ \\
& \quad+\alpha_{1} \int_{\Omega}\left|\boldsymbol{\nabla} f\left(\boldsymbol{p}_{c}^{\varepsilon}\right)\right|^{2} \mathrm{~d} \boldsymbol{\omega}+\alpha_{2} \int_{\Omega}\left|\boldsymbol{\nabla} g\left(\boldsymbol{p}_{c}^{\varepsilon}\right)\right|^{2} \mathrm{~d} \omega+ \\
& \quad+\varepsilon \int_{\Omega} \boldsymbol{\nabla} \boldsymbol{s}_{w}\left(\boldsymbol{p}_{c}^{\varepsilon}\right) \cdot \nabla f\left(\boldsymbol{p}_{c}^{\varepsilon}\right) \mathrm{d} \omega+\varepsilon \int_{\Omega} \boldsymbol{\nabla} s_{g}\left(\boldsymbol{p}_{c}^{\varepsilon}\right) \cdot \nabla g\left(\boldsymbol{p}_{c}^{\varepsilon}\right) \mathrm{d} \omega \leq c_{19}
\end{aligned}
$$

où $c_{19}$ est une constante indépendante de $\varepsilon$.

Le cinquième terme du membre de gauche de l'inégalité (6.10) se majore grâce à l'inégalité de Young par

$$
\frac{2 \varepsilon^{2}}{\alpha_{1}} \int_{\Omega}\left|\nabla s_{w}\left(p_{c}^{\varepsilon}\right)\right|^{2} \mathrm{~d} \omega+\frac{\alpha_{1}}{2} \int_{\Omega}\left|\nabla f\left(p_{c}^{\varepsilon}\right)\right|^{2} \mathrm{~d} \omega .
$$

De même, pour le sixième terme.

En intégrant de 0 à $t$ l'inégalité (6.10) et en tenant compte de l'inégalité ci-dessus, de l'hypothèse $(\mathrm{H} 10)_{4}$ et des estimations (6.8) et (6.9), on déduit

$$
\begin{gathered}
\int_{\Omega} \Phi(x) q\left(p_{c}^{\varepsilon}(t)\right) \mathrm{d} \omega+\frac{\alpha_{1}}{2} \int_{0}^{t} \int_{\Omega}\left|\nabla f\left(p_{c}^{\varepsilon}\right)\right|^{2} \mathrm{~d} \omega \mathrm{d} t+ \\
+\frac{\alpha_{2}}{2} \int_{0}^{t} \int_{\Omega}\left|\nabla g\left(p_{c}^{\varepsilon}\right)\right|^{2} \mathrm{~d} \omega \mathrm{d} t \leq c_{20}
\end{gathered}
$$

où $c_{20}$ est une constante indépendante de $\varepsilon$. 
De cette estimation, on déduit que

$$
f\left(\boldsymbol{p}_{c}^{\varepsilon}\right) \text { et } g\left(\boldsymbol{p}_{c}^{\varepsilon}\right) \text { sont bornées dans } L^{2}([0, t[, \mathcal{V}) .
$$

Rappelons le résultat de J. Simon [17, théorème 3.1].

THÉORÈME DE J. SIMON .- Soit $\Omega$ un ouvert borné de $\mathbb{R}^{N}$, soit un triplet $(\sigma, r, \rho)$ tel que $0<\sigma<1,1 \leq r<\infty, 0<\rho<1$, soit $\varphi$ satisfaisant

$$
\left\{\begin{array}{l}
\varphi \in C^{0}(\Omega) \\
\left|\varphi\left(y^{\prime}\right)-\varphi(y)\right| \leq c\left|y^{\prime}-y\right|^{\rho}, \quad \forall\left(y^{\prime}, y\right) \in \mathbb{R}^{2} .
\end{array}\right.
$$

alors, pour tout $v \in W^{\sigma, r}(\Omega)$

$$
\varphi(v) \in W^{\rho \sigma, r / \rho}(\Omega) \quad \text { et } \quad|\varphi(v)|_{W^{\rho \sigma, r / \rho(\Omega)}} \leq c|v|_{W^{\sigma, r}(\Omega)}^{\rho} .
$$

Remarque 3. - On déduit de ce résultat que si $\varphi \in C^{0}(\Omega)$ et vérifie $\left|\varphi\left(x_{1}, y_{1}\right)-\varphi\left(x_{2}, y_{2}\right)\right| \leq c_{1}\left|x_{1}-x_{2}\right|^{\rho}+c_{2}\left|y_{1}-y_{2}\right|^{\rho}, \quad \forall\left(x_{1}, y_{1}, x_{2}, y_{2}\right) \in \mathbb{R}^{4}$ alors pour tout $(u, v) \in W^{\sigma, r}(\Omega)$,

$$
\varphi(u, v) \in W^{\rho \sigma, r / \rho}(\Omega)
$$

et

$$
|\varphi(u, v)|_{W^{\rho \sigma, r / \rho(\Omega)}} \leq c_{3}|u|_{W^{\sigma, r}}^{\rho}+c_{4}|v|_{W^{\sigma, r}}^{\rho}
$$

On a $L^{2}\left(\left[0, t[; \mathcal{V})\right.\right.$ s'injectant continuement dans $L^{2}\left(\left[0, t\left[; W^{s, 2}\right)\right.\right.$ pour tout $s$ inférieur à un; ainsi de l'estimation (6.11), on a

$$
f\left(\boldsymbol{p}_{c}^{\varepsilon}\right) \text { et } g\left(\boldsymbol{p}_{c}^{\varepsilon}\right) \text { sont bornées dans } L^{2}\left(\left[0, t\left[; W^{s, 2}\right), \quad \forall s \in\right] 0,1[.\right.
$$

D'après l'hypothèse $(\mathrm{H} 10)_{3}$ et le théorème de J. Simon rappelé ci-dessus, on obtient

$$
p_{c w}^{\varepsilon}(t) \text { et } p_{c g}^{\varepsilon}(t) \text { sont bornées dans } W^{\theta s, 2 / \theta}(\Omega) \text { pour presque tout } t,
$$
et de plus on a l'estimation

$$
\left|\boldsymbol{p}_{c \eta}^{\varepsilon}(t)\right|_{W^{\theta s, 2 / \theta}(\Omega)}^{1 / \theta} \leq c_{21}|f(t)|_{W^{s, 2}(\Omega)}+c_{22}|g(t)|_{W^{s, 2}(\Omega)} \quad \text { pour } \eta=w, g .
$$


Par suite en intégrant dans la variable $\tau$ de 0 à $t$ l'inégalité précédente, on a

$$
\left|\boldsymbol{p}_{c \eta}^{\varepsilon}\right|_{L^{2 / \theta}\left(\left[0, t\left[; W^{\theta s, 2 / \theta}(\Omega)\right.\right.\right.}^{2 / \theta} \leq c_{23}|f|_{L^{2}\left(\left[0, t\left[; W^{s, 2}(\Omega)\right.\right.\right.}+c_{24}|g|_{L^{2}\left(\left[0, t\left[; W^{s, 2}(\Omega)\right.\right.\right.} .
$$

Enfin d'après (6.11) et l'estimation ci-dessus, on obtient

$$
\left|\boldsymbol{p}_{c \eta}^{\varepsilon}\right|_{L^{2 / \theta}\left(j 0, t\left[; W^{\theta s, 2 / \theta}(\Omega)\right.\right.} \leq c_{25} \quad \text { pour } \eta=w, g,
$$

où $c_{25}$ est une constante indépendante de $\varepsilon$.

$s_{w}$ et $s_{g}$ sont dérivables de dérivées bornées en $p_{c}^{\varepsilon}$, donc $s_{w}$ et $s_{g}$ sont uniformément lipschitziennes par rapport à $p_{c}^{\varepsilon}$.

Ainsi d'après (6.13), on a

$$
\left|s_{\eta}\left(\boldsymbol{p}_{c}^{\varepsilon}\right)\right|_{L^{2 / \theta}(] 0, t\left[; W^{\theta s, 2 / \theta}(\Omega)\right.} \leq c_{26} \quad \text { pour } \eta=w, g,
$$

où $c_{26}$ est une constante indépendante de $\varepsilon$.

Estimation de $\partial_{t}\left(\boldsymbol{\Phi}(x) \boldsymbol{s}_{w}\left(\boldsymbol{p}_{c}^{\varepsilon}\right)\right)$ et $\partial_{t}\left(\boldsymbol{\Phi}(x) \boldsymbol{s}_{g}\left(\boldsymbol{p}_{c}^{\varepsilon}\right)\right)$

L'équation (6.4) nous permet d'obtenir, $\forall u \in L^{2}([0, t[; \mathcal{V})$,

$$
\begin{aligned}
\left|\left\langle\partial_{t}\left(\Phi(x) s_{w}\left(\boldsymbol{p}_{c}^{\varepsilon}\right)\right), u\right\rangle\right| \leq \\
\leq\left|\nu_{w}\left(s\left(\boldsymbol{p}_{c}^{\varepsilon}\right)\right)\right|_{L^{\infty}(\Omega)}\left|\boldsymbol{V}_{T}^{\varepsilon}\right|_{L^{2}(\Omega)}|\boldsymbol{\nabla} u|_{L^{2}(\Omega)}+ \\
\quad+\left|\mathbf{K}(x) b_{11}\left(\boldsymbol{p}_{c}^{\varepsilon}\right)\right|_{L^{\infty}(\Omega)}\left|\boldsymbol{\nabla} f\left(\boldsymbol{p}_{c}^{\varepsilon}\right)\right|_{L^{2}(\Omega)}|\boldsymbol{\nabla} u|_{L^{2}(\Omega)}+ \\
\quad+\left|\mathbf{K}(x) b_{12}\left(\boldsymbol{p}_{c}^{\varepsilon}\right)\right|_{L^{\infty}(\Omega)}\left|\boldsymbol{\nabla} g\left(\boldsymbol{p}_{c}^{\varepsilon}\right)\right|_{L^{2}(\Omega)}|\boldsymbol{\nabla} u|_{L^{2}(\Omega)}+ \\
\quad+(\operatorname{mes} \Omega)\left|\mathbf{K}(x) \bar{\rho}_{w}\left(s\left(\boldsymbol{p}_{c}^{\varepsilon}\right)\right)\right|_{L^{\infty}(\Omega)}|\boldsymbol{\nabla} u|_{L^{2}(\Omega)}+ \\
\quad+\varepsilon\left|\nabla \boldsymbol{s}_{w}\left(\boldsymbol{p}_{c}^{\varepsilon}\right)\right|_{L^{2}(\Omega)}|\boldsymbol{\nabla} u|_{L^{2}(\Omega)}+ \\
\quad+\left|\nu_{w}\left(s\left(\boldsymbol{p}_{c}^{\varepsilon}\right)\right)\right|_{L^{2}\left(\Gamma_{s}\right)}\left|q_{s}\right|_{L^{2}\left(\Gamma_{s}\right)}|u|_{L^{2}\left(\Gamma_{s}\right)}
\end{aligned}
$$

D'après l'estimation $(6.8)$, on a

$$
\varepsilon\left|\nabla s_{w}\left(p_{c}^{\varepsilon}\right)\right|_{L^{2}(\Omega)} \leq \sqrt{c_{17}} .
$$

Du fait que les saturations soient bornées dans $L^{\infty}\left(\mathbb{R}_{+} \times \Omega\right)$ d'après l'étape 3 , (H4)-(H7) et (6.11), on déduit de (6.15) :

$$
\left|\partial_{t}\left(\boldsymbol{\Phi}(x) \boldsymbol{s}_{w}\left(\boldsymbol{p}_{c}^{\varepsilon}\right)\right)\right|_{L^{2}\left(\left[0, t\left[; \mathcal{V}^{\prime}\right)\right.\right.} \leq c_{27} .
$$


De même de (6.5), on déduit que

$$
\left|\partial_{t}\left(\Phi(x) s_{g}\left(\boldsymbol{p}_{c}^{\varepsilon}\right)\right)\right|_{L^{2}\left(\left[0, t\left[; \mathcal{V}^{\prime}\right)\right.\right.} \leq c_{28}
$$

Afin de prouver la convergence forte de $s\left(\boldsymbol{p}_{c}^{\varepsilon}\right)$ dans $L^{2}\left(\left[0, t\left[; L^{2}(\Omega)\right)\right.\right.$, on a de façon semblable au lemme 1 :

$$
\begin{gathered}
\text { l'injection de } \\
\left\{u \in L ^ { 2 / \theta } \left(\left[0, t\left[; W^{\theta s, 2 / \theta}(\Omega)\right) \mid \partial_{t}(\Phi(x) u) \in L^{2}\left(\left[0, t\left[; \mathcal{V}^{\prime}\right)\right\}\right.\right.\right.\right. \\
\text { dans } L^{2 / \theta}\left(\left[0, t\left[; L^{2 / \theta}(\Omega)\right)\right.\right. \text { est compacte, }
\end{gathered}
$$

qui se déduit du résultat suivant [17] :

l'injection de

$$
\begin{gathered}
\left\{u \in L ^ { 2 / \theta } \left(\left[0, t\left[; W^{\theta s, 2 / \theta}(\Omega)\right) \mid \partial_{t} u \in L^{2}\left(\left[0, t\left[; \mathcal{V}^{\prime}\right)\right\}\right. \text { dans }\right.\right.\right. \\
L^{2 / \theta}\left(\left[0, t\left[; L^{2 / \theta}(\Omega)\right)\right.\right. \text { est compacte. }
\end{gathered}
$$

Par conséquent de $(6.14),(6.17)$ et $(6.18)$, on a

$$
\boldsymbol{s}^{\varepsilon}=\boldsymbol{s}^{\varepsilon}\left(p_{c}^{\varepsilon}\right) \rightarrow s \text { dans }\left(L ^ { 2 / \theta } \left(\left[0, t\left[; L^{2 / \theta}(\Omega)\right)\right)^{2} \text { fortement, } \eta=w, g .\right.\right.
$$

De (6.13), on obtient

$$
\boldsymbol{p}_{c}^{\varepsilon} \rightarrow \boldsymbol{p}_{c} \operatorname{dans}\left(L ^ { 2 / \theta } \left(\left[0, t\left[; W^{\theta s, 2 / \theta}(\Omega)\right)\right)^{2} \text { faiblement }, .\right.\right.
$$

D'une manière identique à l'étape 2 de la section 5 , la relation entre les deux limites $\left(s_{w}, s_{g}\right)$ et $\left(p_{c w}, p_{c g}\right)$ s'écrit

$$
p_{c w}=p_{c w}\left(s_{w}, s_{g}\right) \quad \text { et } \quad p_{c g}=p_{c g}\left(s_{w}, s_{g}\right) .
$$

Enfin en utilisant (6.19) et le fait que $\nu_{\eta}$ et $M$ sont des fonctions continues et bornées en $s_{w}$ et $s_{g}$, le théorème de Lebesgue permet d'écrire pour tout $p$ fini

$$
\begin{aligned}
& \nu_{\eta}\left(\boldsymbol{s}^{\varepsilon}\right) \rightarrow \nu_{\eta}(\boldsymbol{s}) \text { fortement dans } L^{p}\left(\left[0, t\left[; L^{p}(\Omega)\right), p<\infty\right.\right. \\
& M\left(\boldsymbol{s}^{\varepsilon}\right) \rightarrow M(s) \text { fortement dans } L^{p}\left(\left[0, t\left[; L^{p}(\Omega)\right), p<\infty\right.\right. \\
& b_{i j}\left(\boldsymbol{s}^{\varepsilon}\right) \rightarrow b_{i j}(\boldsymbol{s}) \text { fortement dans } L^{p}\left(\left[0, t\left[; L^{p}(\Omega)\right), p<\infty .\right.\right.
\end{aligned}
$$

Compte tenu de (6.11), (6.17)-(6.24), le passage à la limite quand $\varepsilon$ tend vers zéro ne révèle pas de difficulté.

Ceci termine la démonstration du théorème 2. 


\section{Conclusions}

Nous avons donc établi un théorème d'existence de solution faible pour le problème régularisé sous la seule condition de différentiabilité proposée par G. Chavent. Cette condition s'est avérée donner des perméabilités physiquement admissibles [8]. Par contre pour passer à la limite sur le problème régularisé et pour obtenir l'existence de solutions faibles pour le problème dégénéré, nous avons été amené à introduire de nouvelles conditions afin d'absorber la dégénérescence du système. Ces conditions conduisent à des modèles de mobilités et de saturations dont la validité physique n'a pas été étudiée.

En fait lorsque les trois phases sont toujours présentes, le système ne dégénère pas et le passage à la limite peut se faire sans introduire d'hypothèses supplémentaires. Enfin, il est à noter qu'un autre modèle pour l'écriture de la conservation de quantité de mouvement, mieux adapté aux écoulements triphasiques que la loi de Darcy et suggéré par S. Withalker [19], permet d'introduire, dans les équations de conservation de la masse, des termes de diffusions éventuellement non dégénérés. Pour un tel modèle l'existence peut s'obtenir par des techniques analogues à celles utilisées à l'étape 1.

\section{Remerciements}

Les auteurs tiennent à remercier Michel Langlais pour l'intérêt qu'il a porté à ce travail.

\section{Bibliographie}

[1] Alt. (H. W.) et Di Benedetto (E.). - Nonsteady flow of water and oil through inhomogeneous porous media, Annali della Scuola Normale Superiore di Pisa, Série IV, vol. XII, 3 (1985).

[2] Alt. (H. W.) et LucKhaus (S.) . - Quasilinear elliptic-parabolic differential equations, Math. Z. 183 (1983), pp. 311-341.

[3] Amaziane (B.), Bourgeat (A.) et Flamri (H.).- Un résultat d'existence pour un modèle d'écoulements diphasiques dans un gisement à plusieurs types de roches, (à paraître).

[4] Arbogast (T.) . - Two-phase incompressible flow in a porous medium with various non homogeneous boundary conditions, I.M.A., Preprint series 606 (February 1990). 
Existence de solutions faibles pour un modèle d'écoulement triphasique

[5] Brezis (H.) . - Opérateurs maximaux monotones et semi groupes de contractions dans les espaces de Hilbert, North-Holland Mathematics Studies, North-Holland Publishing Comp. 5 (1973).

[6] Chavent (G.) - - A new formulation of diphasic incompressible flows in porous media, Lecture Notes Mathematics, Springer Verlag, 503 (1976), pp. 258-270.

[7] Chavent (G.) et Jaffre (J.) . - Mathematical models and finite elements for reservoir simulation, Studies in Mathematics and its Applications, North-Holland Publishing Comp. 17 (1986).

[8] Chavent G. et Salzano (G.). - Un algorithme pour la détermination de perméabilités relatives triphasiques satisfaisant une condition de différentielle totale, I.N.R.A., Rapport de Recherche 355 (janvier 1985).

[9] FABRIE (P.) . - Solutions fortes et comportement asymptotique pour un modèle de convection naturelle en milieu poreux, Acta Applicandae Mathematicae 7 (1986), pp. 49-77.

[10] Fabrie (P.) et LANGLAIS (M.) . - Mathematical analysis of miscible displacement in porous medium, U.F.R. de Mathématiques et Informatique, Bordeaux I, C.N.R.S. 9008 (1990).

[11] Gagneux (G.) .- Existence et propriétés du temps de percée lors de déplacements forcés eau-huile en milieu poreux, Journal de Mécanique Théorique et Appliqué, vol. 3, 3 (1984), pp. 415-432.

[12] Gagneux (G.), Lefívre (A. M.) et Madaune-Tort (M.).- Une approche analytique d'un modèle black oil des écoulements triphasiques compressibles en ingénierie pétrolière, J. Méca. Théo. et Appl., vol. 6, 4 (1987), pp. 547-569.

[13] Gagneux (G.), Lefínge (A. M.) et Madaune-Tort (M.).- Analyse mathématique de modèles variationnels en simulation pétrolière, Revista Matematica de la Universitad Complutense de Madrid, vol. 2, 1 (1989), pp. 119-148.

[14] Girault (V.) et Raviart (P. A.) . - Finite element methods for Navier Stokes equations, Springer Series in Computational Mathematics, Springer-Verlag 5 (1986).

[15] Hornung (U.). - A parabolic-elliptic variational inequality, Manuscripta Math. 39 (1982), pp. 155-172.

[16] Lions (J.-L.) .- Quelques méthodes de résolutions des problèmes aux limites non linéaires, Dunod, Paris (1969).

[17] Simon (J.) .- Régularité de la composée de deux fonctions et applications, Bolletino U.M.I. (5), 16-B (1979), pp. 501-522.

[18] Simon (J.) . - Compact set in the space $L^{p}(0, T ; B)$, Annali di Matematica Pura ed. Applicata (IV), vol. CXLVI (1987), pp. 65-96.

[19] Withalker (S.) . - Flow in porous media II: The governing equations for immiscible, two-phase flow, Transport In Porous Media 1 (1986), pp. 105-125. 